\title{
A Novel Image-based Ultrasonic Test to Map Material Mechanical Properties at High Strain-rates
}

\author{
R. Seghir ${ }^{1}$ (D) F. Pierron ${ }^{1}$
}

Received: 9 February 2017 / Accepted: 14 August 2017 / Published online: 15 September 2017

(C) The Author(s) 2017. This article is an open access publication

\begin{abstract}
An innovative identification strategy based on high power ultrasonic loading together with both infrared thermography and ultra-high speed imaging is presented in this article. It was shown to be able to characterize the viscoelastic behaviour of a polymer specimen (PMMA) from a single sample over a range of temperatures and strainrates. The paper focuses on moderate strain-rates, i.e. from 10 to $200 \mathrm{~s}^{-1}$, and temperatures ranging from room to the material glass transition temperature, i.e. $110^{\circ} \mathrm{C}$. The main originality lies in the fact that contrary to conventional Dynamic Mechanical Thermal Analysis (DMTA), no frequency or temperature sweep is required since the experiment is designed to simultaneously produce both a heterogeneous strain-rate state and a heterogeneous temperature state allowing a local and multi-parametric identification. This article is seminal in nature and the test presented here has good potential to tackle a range of other types of high strain-rate testing situations.
\end{abstract}

Keywords Ultra-high speed · Visco-elasticity · PMMA . Infrared thermography $\cdot$ Identification

R. Seghir

r.seghir@soton.ac.uk

F. Pierron

f.pierron@soton.ac.uk

1 Faculty of Engineering and the Environment, University of Southampton, Southampton, UK

\section{Introduction}

In many instances in life, materials around or within us suffer deformation at high rates. This is the case when engineering structures undergo impact, crash, blast, etc. but also when forming materials like stamping or machining for instance. Another important area concerns biological tissues. For instance, traumatic brain injuries (TBI) involve damage of brain tissues caused by their high rate deformation following impact load of the skull. Thanks to the significant progress in computing power and computational mechanics tools, it is now possible to perform extremely detailed numerical simulations of many complex situations where materials deform at high rates, with the objective to design safer structures, assess tissue injuries or devise more effective manufacturing processes, as mentioned above. However, to deliver their full potential, these computations require the input of reliable and accurate mechanical constitutive models of the materials loaded at high strain-rates. This is an extremely challenging problem because of both the dynamic nature of the mechanical fields and the technological difficulties associated with strain metrology. Presently, this represents an important scientific bottleneck for society to fully benefit from such advances in numerical simulation.

A number of testing techniques are available to identify the high strain-rate properties of materials, as reviewed in [5]. Most of them rely on very limited experimental information, such as strain gauges or point velocity measurements with VISAR technology. As a consequence, the tests need to follow strict assumptions to relate the measurements to the material behaviour, for instance, uniform strain field and no inertia effects are typical assumptions in split Hopkinson pressure bar (SHPB) testing. 
The advent of full-field optical metrology, such as digital image correlation [35] or the grid method [10], combined with the new developments in ultra-high speed imaging $[2,29,36]$ provides a unique opportunity to revisit high strain-rate testing techniques. In particular, full-field of accelerations can be obtained which provides a powerful image-embedded load cell if the material density is known, which is usually the case. This concept was first proposed in 2009 in [22], published in full in 2011 [23], where a proof of principle experiment was performed on quasi-isotropic composite specimens, with and without a free hole. The in-plane Young's modulus and Poisson's ratio were identified satisfactorily before the onset of damage, within the first $30 \mu \mathrm{s}$ of the test, at strain-rates up to $1000 \mathrm{~s}^{-1}$. The grid method was used to measure the deformation together with a Cordin 550-62 rotating mirror ultra-high speed camera operating at $30 \mathrm{kfps}$. However, this test was performed on a tensile Kolsky bar, though the bars were not used to obtain the impact force. The concept was then extended to purely inertial tests which showed to be much more suited for this kind of analysis. The very first example dealt with concrete spalling tests [26], and was then extended to composites [27] and metals [3, 4, 19]. Since then, the idea has spread and several groups worldwide are starting to use the technique [13, 14, 16, 17, 21, 40, 41].

Initially, this idea to use the full-field acceleration as a load cell with the Virtual Fields Method came from seminal work by Prof. Michel Grédiac [8, 9] using vibration tests. In this case, high speed imaging is not necessary and the acceleration derives easily from the deflection using the harmonic assumption. This was extended later on to include damping [7]. Recently, an article showed that ultrasonic excitation combined with ultra-high speed and infrared imaging [37] could be used to image the high strain-rate deformation of a polymeric foam, though the authors did not use the acceleration to identify stiffness. This was released in a later contribution [25]. The present paper builds up on this to explore the potential of this test in more depth on a homogeneous PMMA specimen. In particular, the original idea of the paper consists in investigating how heterogeneous loadings states, e.g. stationary or transient deformation waves, combined with the measurement of the local strain, strainrate, temperature and stress (from acceleration) states can be used to identify viscoelastic material properties over a wide range of thermo-mechanical conditions. In other words, this provides a unique opportunity to identify from one single experiment, data which would have normally required a battery of tests and samples. This work is seminal in nature and focuses on the methodology rather than on the analysis of the material behaviour. However, comparison with literature results and Dynamic Mechanical Thermal Analysis (DMTA) performed on a specimen from the same material sheet enables to gain confidence in the obtained results. In addition the present data concern the moderate strain-rate range, tens to hundreds of $s^{-1}$, which is notoriously difficult to access with conventional servo-hydraulic machines or SPHB.

In a first part, the experimental setup and the theoretical framework are detailed. In a second part, the experimental results are presented and discussed. Finally, the identification process is simulated to provide a first idea of the optimal experimental parameters required to achieve a precise identification.

\section{Experiment and Data Processing}

\section{Experimental Setup}

The concept of the experiment consists in producing within a viscoelastic sample, both a heterogeneous deformationrate state and a heterogeneous temperature state, and capturing the thermo-mechanical response of the material through full-field measurements. To achieve this, five key elements are required, as shown in Fig. 1.

A high-power ultrasonic transducer - The NextGen Lab 750 system from SynapTec (France) allows to cyclically deform the sample at $20 \mathrm{kHz}$ up to a peak-to-peak displacement amplitude of $120 \mu \mathrm{m}$, depending on the sample design and damping. The actuator is a bolt-clamped Langevin type transducer composed of a stack of piezoelectric elements resonant at $20 \mathrm{kHz}$. The displacement amplitude is boosted using a titanium horn together with the transducer, i.e. a mechanical amplifier. The output horn diameter, i.e. the active surface, is $12 \mathrm{~mm}$, so that samples up to the same width can be attached to it.

A middle wavelength ${ }^{1}$ infrared (IR) camera - The Silver 480M from Cedip (now FLIR) measures the heat flux radiated by the deforming sample and reconstructs space resolved surface temperature fields. The IR camera has a resolution of $320 \times 256$ pixels, a NEDT ${ }^{2}$ of about $20 \mathrm{mK}$ at $25{ }^{\circ} \mathrm{C}$, and 6000 frames can be recorded up to $383 \mathrm{~Hz}$. Reducing the acquisition window down to $64 \times 12$ pixels allows to reach a frame rate of $16 \mathrm{kHz}$ [6]. Nevertheless, in the present experiment, the camera was used at a frame rate of 50 fps only, i.e. 1 frame every 400 loading cycles at 20 $\mathrm{kHz}$, with an integration time of $509 \mu \mathrm{s}$ to cover the range $20-100{ }^{\circ} \mathrm{C}$. The low frame rate was chosen to record the temperature over a long time frame, this point is detailed later on. Finally, the camera was used together with a $27 \mathrm{~mm}$ lens leading to a working distance of about $140 \mathrm{~mm}$ and a field of view of $60 \times 48 \mathrm{~mm}$. An example of an IR frame captured by the camera is shown in Fig. 2(b). The sample

\footnotetext{
13-5 $\mu \mathrm{m}$.

${ }^{2}$ Noise equivalent differential temperature.
} 


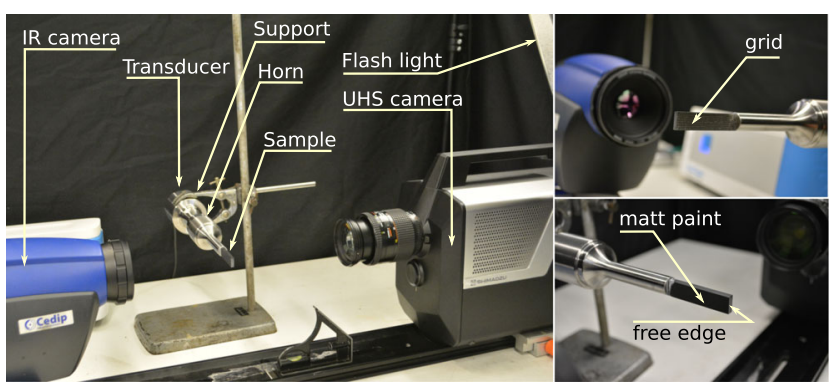

Fig. 1 Image-based DMTA experimental setup. Sample dimensions are $52 \mathrm{~mm} \times 12 \mathrm{~mm} \times 4 \mathrm{~mm}$

is delineated by the black dotted line, its free edge is on the right while the tip of the ultrasonic horn can be seen on the left.

An ultra-high speed (UHS) camera - The HyperVision HPV-X camera from the Shimadzu Corporation (Japan) is used to capture images of the deforming sample to obtain space and time resolved displacement and acceleration ${ }^{3}$ fields. The UHS camera has a resolution of $400 \times 250$ pixels and 128 frames can be recorded up to a frame rate of $5 \mathrm{Mfps}$. Here, a frame rate of $500 \mathrm{kfps}$ was chosen, i.e. 25 frames/cycle at $20 \mathrm{kHz}$. The sensor noise was measured on stationary images to be in the order of $3.5 \%$ of pixel dynamic range. Details concerning the methodology used to obtain this value are provided in "Measurement Uncertainty". The specimen was illuminated by a Gemini 1000Pro (1000 W) flash light from Bowens (UK). An example of a frame captured by the camera is shown in Fig. 2(a). The magnification was chosen to fit at best the sample length, the free edge is on the left part of the frame.

A bonded regular grid was used to extract the in-plane displacement fields through a phase-shifting algorithm [10]. The grid, from COLOURSENSE Ltd. (UK), was produced using the dry transfer technique, i.e. by outputting a vector artwork to a high resolution film negative and using a photo-lithographic technique to expose a carrier sheet coated with a photosensitive ink-based film deposited on top of a thin layer of glue. The resulting pressure-sensitive adhesive multi-layer was carefully aligned on the sample surface, manually pressed, and the carrier sheet simply peeled-off. The total thickness of the grid has been measured to be less than $100 \mu \mathrm{m}$, typically around $40-70 \mu \mathrm{m}$. It was demonstrated in [28] that an equivalently thick layer of epoxy glue did not disturb the strain measurements when compared to a strain gauge. The grid used in the present configuration had a pitch of $1 \mathrm{~mm}$ and was imaged through a $28-105 \mathrm{~mm}$ Nikkor lens, at a sampling of 7 pixels per period, allowing a field of view of $57 \times 35 \mathrm{~mm}$ with a working distance of about $180 \mathrm{~mm}$ (see Fig. 1).

\footnotetext{
${ }^{3}$ After double temporal differentiation.
}

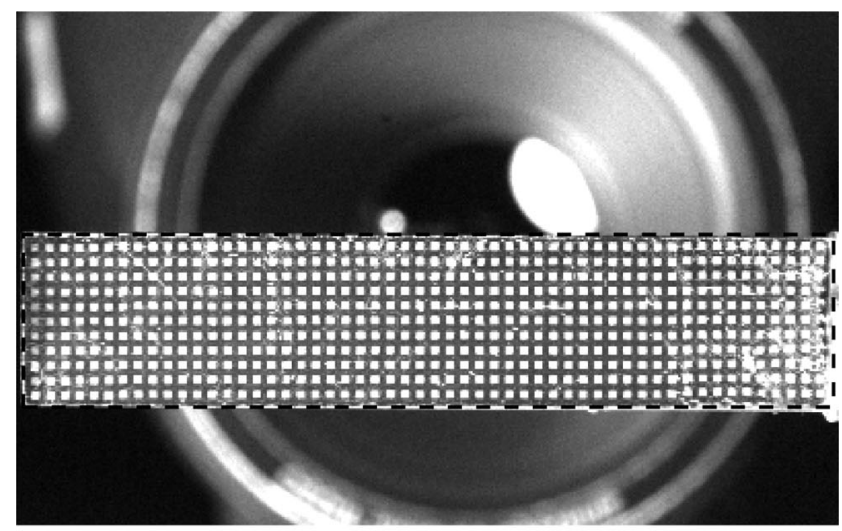

(a) Example of an image of the sample at rest, from the UHS camera. The grid pitch is $1 \mathrm{~mm}$. The lens of the IR camera can be seen in the background.

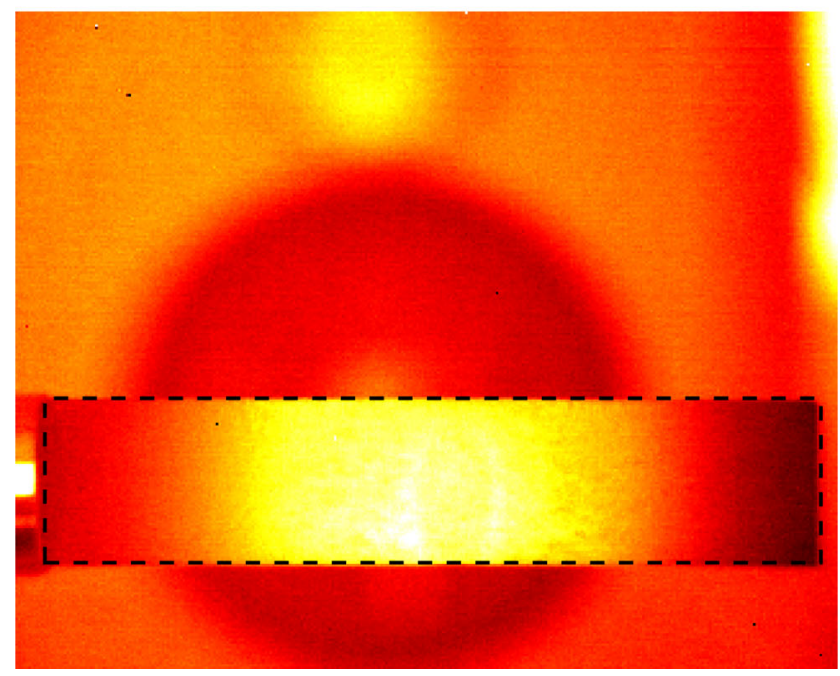

(b) Example of a temperature field captured during an ultrasonic run. The temperature rises due to the self-heating process can be seen at the centre of the sample while the sample left and right edges remain close to room temperature. The lens of the UHS camera can be seen in the background.

Fig. 2 Example of grabbed images. Sample dimensions are $52 \mathrm{~mm} \times$ $12 \mathrm{~mm}$, with a thickness of $4 \mathrm{~mm}$

A sample The material chosen for this first investigation was a $4 \mathrm{~mm}$ thick, $55 \mathrm{~mm}$ long and $12 \mathrm{~mm}$ wide PMMA (Poly-Methyl-MethAcrylate) Acrycast ${ }^{\circledR}$ sample sourced from Amari Plastics Plc (UK). The sample length was chosen to ensure that its first longitudinal deformation mode was at $20 \mathrm{kHz}$. Quasi-static reference material properties were obtained at $\dot{\varepsilon}=10^{-2}$ in uni-axial stress configuration using a standard universal test machine. Back-to-back strain gauges were employed to take into account spurious bending. A tangent Young's modulus value of $E=2.9^{ \pm 0.1}$ GPa and a Poisson's ratio value of $v=0.35^{ \pm 0.01}$ were found. The material density was obtained by measurement of water displacement to a value of $\rho=1160 \mathrm{~kg} \cdot \mathrm{m}^{-3}$. In addition, the glass transition temperature $\mathrm{T}_{g}$ of the material has been measured using both DMTA and Differential Scanning Calorimetry (DSC), and found to be about $110^{ \pm 5}{ }^{\circ} \mathrm{C}$. 
DMTA data has been obtained from a Q800 analyser from TA Instruments using the dual cantilever (bending) deformation mode at a loading amplitude and a frequency of 0.1 $\%$ and $1 \mathrm{~Hz}$ respectively and using a temperature ramp of $1{ }^{\circ} \mathrm{C} \cdot \mathrm{min}^{-1}$. In the present work, simultaneous deformation and IR temperature measurements are required. To achieve this, the sample was covered with a black matt paint on one face, to produce a uniform and high emissivity surface suitable for IR measurements and, on the other face, with the regular grid described above (see Fig. 1).

\section{Experimental Procedure and Data Processing}

The experiment consisted in a set of successive short ultrasonic runs during which both IR and UHS images were recorded. A schematic representation of the procedure is provided in Fig. 3. The ultrasonic generator was programmed to produce, every $15 \mathrm{~s}$, a $800 \mathrm{~ms}$ amplitude controlled cyclic loading and to send a trigger to the UHS camera. The flash light was triggered by the camera and a $256 \mu \mathrm{s}$ image sequence of the deforming sample was recorded. The camera buffer was then automatically emptied by transferring the images to the computer and then re-armed for the next run. This operation lasts about $5 \mathrm{~s}$ depending on the computer and the connection speed. In parallel, the IR data were continuously recorded to capture the entire thermal history over a certain number of ultrasonic runs and for different loading amplitude. Indeed, this procedure can be applied for different loading displacement amplitudes, to produce sets of tests covering different strain-rate, temperature and heat-rate values. In the present paper, the procedure presented in Fig. 3 was performed twice on a single sample, first at low displacement amplitude $( \pm 15 \mu \mathrm{m})$, then at higher amplitude $( \pm 25 \mu \mathrm{m})$ in an attempt to cover more of the strain-rate/temperature space.

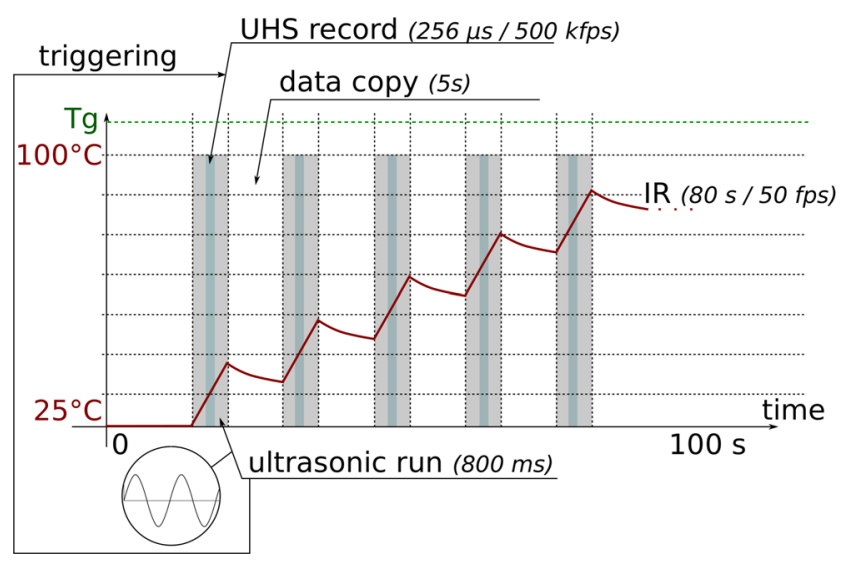

Fig. 3 Principle of the test
The $2^{\text {nd }}$ series of test was conducted until the material locally reached its glass transition temperature (see Fig. 3). All these points will be discussed later on in the article.

To summarize, the data set consisted of sets of discrete grid images sequences and continuous recordings of the surface sample infrared radiation. A schematic representation of the entire data post-processing procedure is provided in Appendix. As introduced above, displacements were obtained from grid images using the grid-method [10]. In practice, initial and current image phases, $\phi_{x}$ and $\phi_{y}$, were calculated individually through a windowed discrete Fourier transform (WDFT) algorithm using a triangular windowing of $2 N-1$ size, $N$ being the grid sampling (number of pixels per period). The displacements were then obtained from the spatial phase shifts between a reference (first captured frame) and the following images according to the following equation:

$U_{i}(x, y, t)=\frac{p}{2 \pi} \Delta \phi_{i}(x, y, t)$, with $i=(x, y)$

where $U_{i}$ is the displacement field along the direction $i$, $\Delta \phi_{i}$ is the phase shift along the same direction and $p$ is the grid pitch. Because of the $2 \mathrm{~N}-1$ kernel for the WDFT algorithm, one grid pitch is systematically lost at edges. As the whole displacement field is required for the data processing, especially at the sample free edge, missing data points were reconstructed using cubic spline extrapolation. Displacement fields were smoothed using a Gaussian filter (in space and time) before calculating derivatives and the optimal smoothing kernel size has been found to be $7 \times 7 \times$ 7 along the $\mathrm{x}, \mathrm{y}$ and time directions. Information concerning the choice of the smoothing kernel size are provided in "Finite Element Validation". The final post-processing operation on the displacement consisted in finding and subtracting the initial deformation state. The choice of the initial undeformed reference image needs to be commented on at this stage. Indeed, stationary images were recorded before the start of each series of tests. However, because of the heating up of the specimen, thermal strains build up with time and the use of the unloaded image as a reference means that the strain fields will contain both thermal and mechanical strains, while only the later are of interest here. To avoid this issue, the first image of each ultrasonic run has been used as a reference image. This however leads to another issue. Indeed, as presented in Fig. 3, for each run the first captured frame does not necessarily correspond to the sample at rest (ie, $t=0$ in the harmonic response). Therefore, displacement fields based on such a reference need to be corrected. The fact that the loading is harmonic means that the average displacement of any material point over a period should be null. Such an assumption allows the 
identification of an offset field, i.e. the point by point average of the displacement over 5 periods, which is then subtracted from the initially estimated displacement. Finally, the displacements were averaged along the sample width as the test is assumed to be 1D. Strain, strain-rate and acceleration fields are subsequently simply obtained through simple and double centred finite difference while derivatives at sample boundaries are obtained through left- and right-hand side finite difference respectively. It is worth noting that acceleration fields can also be obtained through analytic differentiation as follows:

$\tilde{a}_{i}(x, t)=-\omega^{2} U_{i}(x, t)$

with $\omega=2 \pi f$ the angular frequency. The systematic comparison between analytic derivation (see Equation (2)) and acceleration obtained from finite differences will be discussed within "Finite Element Model", dedicated to FE simulations, but finite differences only were used for experimental data-processing in "Results and Discussion". This point will be discussed later on.

Infrared (IR) data were post-processed following the socalled pixel-by-pixel calibration strategy [34]. Sets of 20 IR reference images at different uniform and stabilized temperatures, from 20 to $90^{\circ} \mathrm{C}$, were captured using an extended area blackbody from Infrared System Development Corp. (USA). The mean response of each pixel of the IR focal plane array (FPA) as a function of the blackbody temperature was then fitted using a $5^{\text {th }}$ order polynomial function to build up a set of coefficients which fully characterise the relationship between the raw digital level and the effective temperature for each pixel and over the expected experimental temperature range. This calibration was then applied to the experimental measurements to convert the raw data into temperature fields. As kinematic measurements have been obtained over discrete $256 \mu$ s periods while infrared data were continuously recorded at $50 \mathrm{fps}$, the next step consists in finding the single measured thermal field corresponding at best to each kinematic sequence. In the present work, the thermal field taken at time nearest to half the loading period was simply selected. The impact of this choice on the accuracy of the thermal measurement regarding the considered kinematic sequence is discussed in the next section ("Measurement Uncertainty"). Finally, the selected thermal fields were downscaled using spline interpolation to fit the kinematic measurements resolution, and then, averaged over the sample width.

The procedure for reconstruction of stress fields and material identification will be detailed further in "Theoretical Framework" and "Storage modulus and damping identification".

\section{Measurement Uncertainty}

The sources of uncertainty on the kinematic measurement are multiple. They can arise from the intrinsic sensor noise, the method used to recover displacements, the test conditions etc... This uncertainty can be partly quantified by grabbing a set of stationary undeformed images and applying the phase shifting algorithm [10] to recover displacements, and post-process these displacements (see "Experimental Procedure and Data Processing") to derive strains, strain-rates and accelerations. The resulting identified noise takes into account all these potential sources of error, at least in static conditions. Unfortunately, it does not include errors arising from a misalignment of the sample with respect to the camera reference system and lens distortions which can create some fictitious deformations. It also does not take into account lighting variations and grid defects. Therefore, it can be considered a lower bound of the deformation uncertainty. It should also be noted that the discretized deformation measurement acts as a low pass spatial filter. The systematic error generated by this is explored in "Synthetic Grid Deformation".

Concerning the infrared measurements, the uncertainty is mainly driven by five parameters: (1) the intrinsic noise of the sensor under perfect conditions, i.e. the NEDT over the studied temperature range - (2) the accuracy of the calibration procedure - (3) the surface emissivity distribution and variations - (4) the setup environment and (5) the triggering mismatch. The impact of the environment and emissivity distribution has been mitigated by applying a high emissivity uniform coating to the sample and covering the entire setup with a black curtain during the whole test to avoid IR reflections from the surroundings. The thickness of the coating can also be a problem since it tends to delay the transmission of the thermal information from the specimen to the paint surface [31]. However, this has not been investigated here as the accuracy requirements for the temperature readings are not as stringent as for thermal stress analysis as used in [31]. As detailed in "Experimental Procedure and Data Processing", the calibration was performed using a pixel-by-pixel calibration to minimize the uncertainty. The last point is not exactly a problem of temperature uncertainty but relates to the synchronization of the kinematic and thermal data. Indeed, the temperature was recorded at a lower frame-rate than the kinematic data, and no synchronization between the UHS and IR cameras had been implemented. One can decompose this uncertainty as the sum of: (1) the unknown temperature variation during the few cycles captured by the UHS camera - less than $5 \mathrm{mK}$ - (2) the temperature variation between 2 IR images - about $500 \mathrm{mK}$ and (3) the unknown concerning the precise time when the UHS frames were grabbed with respect to the IR timeline 
- less than $5^{\circ} \mathrm{C}$. The value provided depends on the material self-heating rate and is therefore only valid for the experimental conditions described above (see "Experimental Procedure and Data Processing"). It is worth noticing that the last point is not an intrinsic problem of the method and will be solved in further experiments. However, it is important to keep in mind that the $5{ }^{\circ} \mathrm{C}$ uncertainty in the present case is acceptable as the material temperature sensitivity remains low over this temperature range.

Table 1 summarizes the experimental parameters and reports the related uncertainties. These were calculated from a set of 128 images of the stationary specimen recorded in the same conditions as that of the test just before the start of the ultrasonic horn.

- The grey level noise was obtained by calculating the standard deviation over time at each pixel, and dividing it by the mean grey level value at that pixel. This was then averaged across all grey level values. The sensor noise variation as a function of the sensor dynamic range is discussed in "Synthetic Grid Deformation".
- The strain, strain-rate and acceleration noise values were obtained from the standard deviation in space and time of the average value across $y$ (as the strain will be obtained for the stress-strain curves).

- The stress noise was calculated from the acceleration noise by averaging over the length and multiplying by the density, as in Equation (3).

\section{Theoretical Framework}

This section describes how acceleration maps can be used to derive stress information using a simplified version of the dynamic equilibrium equation. The extent of possible loading ranges accessible with such a setup is then explored.

\section{Acceleration as a load-cell}

As already shown in [25, 27], it is possible to reconstruct average stress distributions along the length of the sample from
Table 1 Experimental parameters and uncertainties obtained from stationary images

\begin{tabular}{|c|c|c|}
\hline \multirow[t]{3}{*}{ Sample } & Material & PMMA \\
\hline & Dimensions (mm) & $55 \times 12 \times 4$ \\
\hline & Density $\left(\mathrm{kg} \cdot \mathrm{m}^{-3}\right)$ & 1160 \\
\hline \multirow[t]{3}{*}{$\left(\dot{\varepsilon}=10^{-2}\right)$} & Young's modulus (GPa) & $2.9^{ \pm 0.1}$ \\
\hline & Poisson's ratio & $0.35^{ \pm 0.01}$ \\
\hline & $\mathrm{T}_{g}\left({ }^{\circ} \mathrm{C}\right)$ & $110^{ \pm 5}$ \\
\hline \multirow[t]{3}{*}{ Grid } & Thickness $(\mu \mathrm{m})$ & $<100$ \\
\hline & Grid pitch (mm) & 1 \\
\hline & Sampling (pixel.period ${ }^{-1}$ ) & 7 \\
\hline \multirow[t]{7}{*}{ IR } & Model & CEDIP Silver 480M \\
\hline & Lens & $27 \mathrm{~mm}$ \\
\hline & Frame rate (fps) & 50 \\
\hline & Integration time $(\mu \mathrm{s})$ & 509 \\
\hline & Number of pixel (pixel²) & $320 \times 256$ \\
\hline & Field of view $\left(\mathrm{mm}^{2}\right)$ & $60 \times 48$ \\
\hline & Uncertainty & $<5^{\circ} \mathrm{C}$ \\
\hline \multirow[t]{13}{*}{ UHS } & Model & Shimadzu HPV-X \\
\hline & Digitization & $10-$ bit \\
\hline & Lens & $28-105$ mm Nikkor \\
\hline & Frame rate $(\mathrm{kfps})$ & 500 \\
\hline & Number of pixel $\left(\right.$ pixel $\left.^{2}\right)$ & $400 \times 250$ \\
\hline & Max. sensor noise ( $\%$ of grey levels) & 3.5 \\
\hline & Field of view $\left(\mathrm{mm}^{2}\right)$ & $57 \times 35$ \\
\hline & Smoothing window $(\mathrm{x}, \mathrm{y}, \mathrm{t})$ & $7 \times 7 \times 7$ \\
\hline & Displacement noise & $0.6 \mu m$ or $5 \times 10^{-3}$ pix \\
\hline & Strain noise (Finite diff. in $\mu$ def) & \pm 195 \\
\hline & Strain-rate noise (Finite diff. in $\mathrm{s}^{-1}$ ) & \pm 25 \\
\hline & Acceleration noise (Finite diff. in $\mathrm{m} . \mathrm{s}^{-2}$ ) & $\pm 1.3 \times 10^{4}$ \\
\hline & Stress noise $(\mathrm{MPa})$ & \pm 0.15 \\
\hline
\end{tabular}


the following relationship (obtained from basic dynamic equilibrium considerations):

$\overline{\sigma_{x x}}(x, t)=-\rho x \overline{a_{x}}(x, t)$

where $\overline{\sigma_{x x}}(x, t)$ is the average Cauchy stress over the transverse section coordinate $y, \rho$ is the material density, and $\overline{a_{x}}(x, t)$ is the surface average of the longitudinal acceleration component between the free edge, and the considered section of coordinate $x$ (see Fig. 4). Such an equation relies on two main assumptions: the homogeneity of the acceleration through the thickness and the homogeneity of the material density over the whole sample. For the latter however, it is possible to update the density using the strain information [15]. Since strains are also obtained by spatial differentiation of the displacements, an assumption of uniaxial loading means that stress-strain curves can be directly reconstructed in many sections of the sample which see different strain, strain-rate and temperature levels.

One understands here that the stress reconstruction precision will depend on three main elements: (1) the accuracy of the material density value which plays the role of load cell factor here, (2) the temporal resolution of the grey level images which will affect the acceleration and (3) the spatial resolution as Equation (3) stems from discrete approximations of spatial integrals [25, 27]. In practice, an additional issue arises from the fact that strains are obtained on average over a grid pitch and expressed in its centre, while stresses are obtained on a slice. An additional step consisting in interpolating the stress values at grid centroids will be required to produce consistent stress-strain curves and identified material properties.

\section{High-power ultrasonic excitation}

One of the key elements of the present methodology is the ability to produce a heterogenous thermo-dynamical state within the material. Such state could be achieved using different strategies, for instance playing on the sample geometry and/or on the loading itself. The choice here

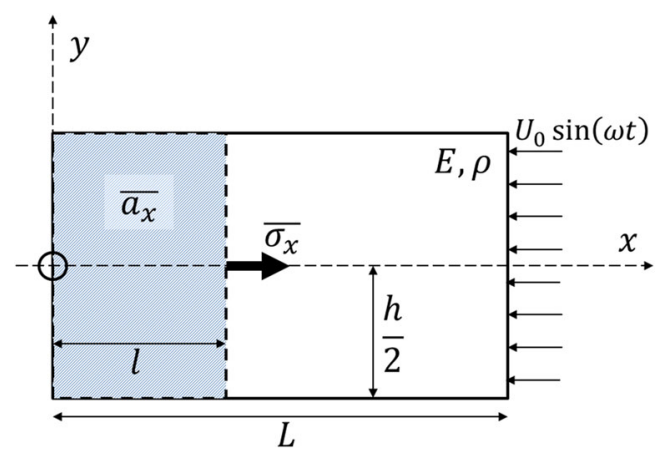

Fig. 4 Schematic of a sample subjected to longitudinal inertial loading is to develop longitudinal stationary waves within the sample. The frequency, the wavelength and the amplitude of such deformation waves will drive the characteristics of the loading.

Let us assume that the displacement field within the sample can be the described as the product of a spatial and temporal sine function such as:

$u(x, t)=u_{0} \cos \left(\omega^{s} x-\Phi^{s}\right) \cos \left(\omega^{f} t-\Phi^{f}\right)$

where:

- $u_{0}$ is the amplitude of loading

- $\omega^{s}=\frac{2 \pi}{L_{n}}$ is related to the spatial deformation wave, with $L_{n} \approx \frac{1}{f} \sqrt{\frac{E}{\rho}}$ the deformation wavelength ( $f$ the loading frequency) and $\sqrt{\frac{E}{\rho}}$ the speed of sound in the material;

- $\quad \omega^{f}=2 \pi f$ is the angular frequency, with $f$ the loading frequency;

- $\Phi^{s}$ and $\Phi^{f}$ are the spatial and temporal phases.

With this description, it is possible to evaluate the levels of maximum strain, strain-rate and acceleration that can be achieved with the current setup:

$$
\begin{aligned}
& \left|\varepsilon_{x}^{\max }\right|=\left(\frac{\rho}{E}\right)^{\frac{1}{2}} u_{0} \omega_{f} \\
& \left|\dot{\varepsilon}_{x}^{\max }\right|=\left(\frac{\rho}{E}\right)^{\frac{1}{2}} u_{0} \omega_{f}^{2} \\
& \left|a_{x}^{\max }\right|=u_{0} \omega_{f}^{2}
\end{aligned}
$$

Three main parameters drive the deformation amplitude: the amplitude of the displacement and the loading frequency, both imposed by the actuator, and the deformation wavelength arising from the material itself.

As a consequence, reaching a strain-rate of e.g. $200 \mathrm{~s}^{-1}$ at a frequency of $20 \mathrm{kHz}$ (standard frequency for high power ultrasonics) requires a peak-to-peak amplitude of $20 \mu \mathrm{m}$ for a PMMA sample $\left(\rho=1200 \mathrm{~kg} \cdot \mathrm{m}^{-3}, E=3\right.$ $\mathrm{GPa}$ ). In other words, covering a large strain-rate domain requires high-power ultrasonics, i.e. both high amplitude and high frequency. This is generally opposed to low-power ultrasound (hundreds of nanometers) which are used for diagnostics and control and does not significantly affect the environment in which the wave propagates. Keeping the values given above, the associated strain amplitude would be of the order of $\pm 0.2 \%$, without taking into account any thermal effects, and the acceleration would reach $320 \mathrm{~km} . \mathrm{s}^{-2}$, i.e. $>3 \times 10^{4} \mathrm{~g}$. Spatially, if the sample is designed to be resonant at its first longitudinal mode, i.e. the sample length is half the wavelength, the sample undergoes high displacement and zero strain on free and fixed edges (see Fig. 4) and high strain and strain-rate at its centre. In addition, due to the 
viscoelastic dissipation, the central part of the sample will heat up cycle after cycle, while the edges will remain almost at room temperature. The last point is due to the significant mismatch between the characteristic conduction time within a PMMA sample [12] and the loading frequency, providing adiabatic conditions during loading.

The above shows that it is possible to reach large strainrates in the material, of the order of hundreds of $s^{-1}$, with a heterogeneous state of strain, strain-rate and temperature which enables to test the material over a wide range of thermo-mechanical conditions within a single test. The present article aims at demonstrating the experimental feasibility of this idea and at providing initial results on PMMA.

\section{Results and Discussion}

\section{Presentation of the Results}

A single PMMA sample was submitted to the experimental procedure detailed above (see "Experimental Procedure and Data Processing"). Successive sequences of grey level images were captured while the temperature was continuously recorded until the sample reached its glass transition temperature. The test was composed of two series of successive ultrasonics runs. In the first series, six ultrasonic runs were successively applied to the sample at low power, leading to a maximum strain of $\pm 735 \mu$ def. It was then followed by five other runs at a higher actuator power, leading to a maximum strain of $\pm 1542 \mu \mathrm{def}$. Between both test series, the sample was allowed to cool down back to room temperature.

Figure 5 presents the temperature record at the highest power ( $2^{\text {nd }}$ series). The red lines show the different instants when short ultrasonic runs were applied (shaded region in Fig. 3) and the map shows how the sample temperature profile (averaged over the sample width) evolves as a function of time. During each loading ( $800 \mathrm{~ms})$, the sample rapidly heated up (within the red line) and then cooled down until the next run. Figure 5 shows this cooling process with a slight decrease in temperature between two red lines. Only $4 \mathrm{~s}$ before and after each ultrasonic loading are shown on the figure. Black dots symbolize the skipped IR frames.

Figure 6 shows the evolution of the measured longitudinal displacement, strain, strain-rate and reconstructed stress profiles as a function of time. The data corresponds to the first ultrasonic run at higher strain amplitude ( $2^{\text {nd }}$ series $)$, i.e. within the first red line in Fig. 5. As stated in "Experiment and Data Processing", the data at a given time step is represented as a 1D signal, therefore, Fig. 6 shows space vs time plots. These maps evidence that the sample is indeed loaded on its first longitudinal deformation mode, since

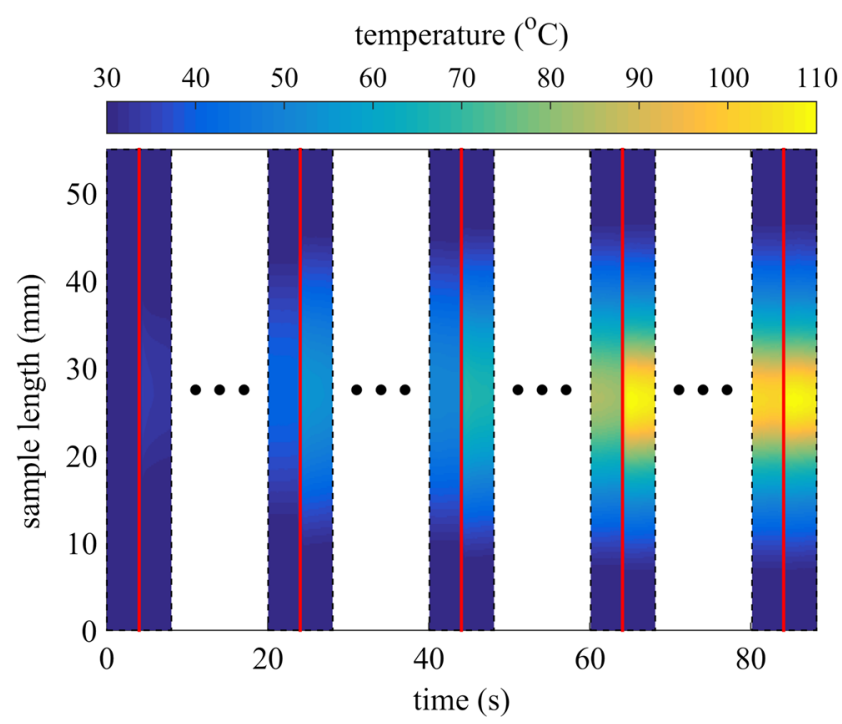

Fig. 5 Temperature profiles (along sample length) as a function of time during the second ultrasonic test series. Red lines are loading instants while the black dots are used to symbolize the delay between loading instants

stationary and half wavelength waves can be observed during the $256 \mu$ s captured by the camera. The sample undergoes a cyclic displacement of $\pm 25 \mu \mathrm{m}$, cyclic deformation of $\pm 1500 \mu$ def, cyclic strain-rate of about \pm 200 $\mathrm{s}^{-1}$ and a cyclic stress of about $\pm 7 \mathrm{MPa}$. Regarding Fig. 5, the sample undergoes also a local increase of temperature up to $110^{\circ} \mathrm{C}$ at the end of the test while the sample edges remains close to room temperature. The following thermomechanical fields are consistent with the analytic estimates presented in "High-power ultrasonic excitation" and confirm the heterogeneity of the thermodynamical state within the material.

Going down into the detail of each ultrasonic run, Fig. 7 presents the strain, strain-rate and stress amplitude profiles (half peak-to-peak) as well as the temperature profiles for every successive ultrasonic loading. Such profiles are computed by calculating, for each sample section, half of the peak-to-peak amplitude over 5 ultrasonic cycles (see Fig. 6) for each of the 11 successive ultrasonic runs. Apart from visualisation, the following data is used to define the value of the apparent strain-rate per sample section. Indeed, each section undergoes a cyclic load and thus, a range of strainrates. Nevertheless, as it is usually assumed in standard Dynamic and Mechanical Analysis, for a sake of simplicity, the time variation can be collapsed into a single apparent strain-rate value defined here as half the amplitude of strain-rate seen by each section. The consequences of this assumption are discussed further in "Outstanding Issues and Scope of The Method". Finally, the frame-rate of the IR camera does not allow a temporal description of the temperature field over each cycle. As a consequence, each 
Fig. 6 Displacement, strain, strain-rate and stress as a function of time. The data have been averaged across the specimen width. The data corresponds to the $1^{\text {st }}$ vertical red line in Fig. 5

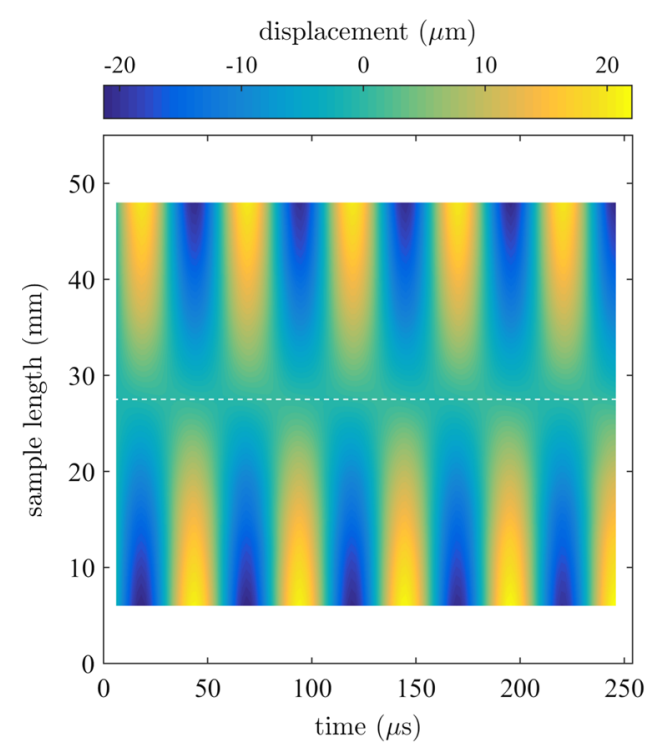

(a) displacement strain-rate $\left(\mathrm{s}^{-1}\right)$

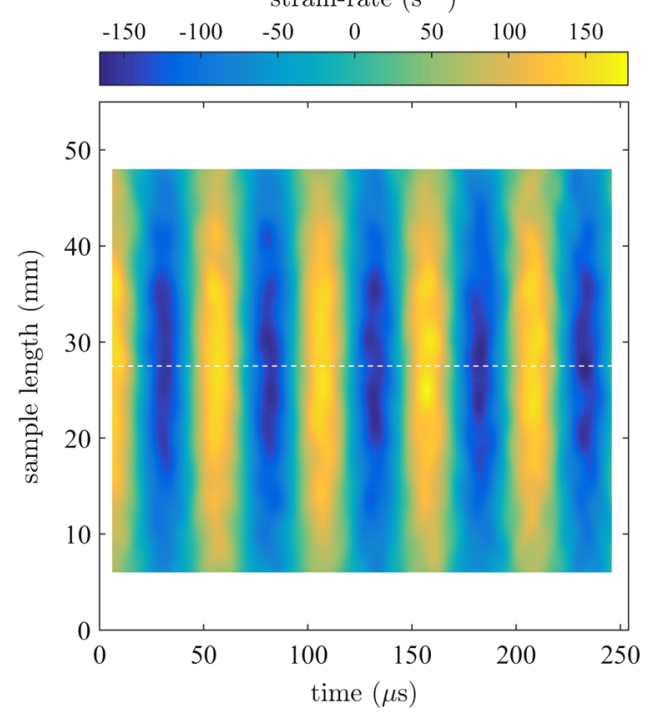

(c) strain-rate

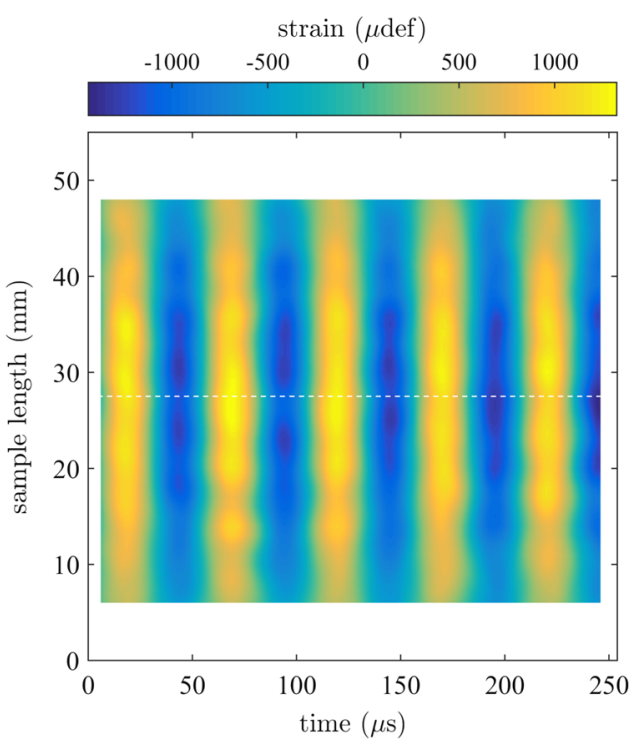

(b) strain

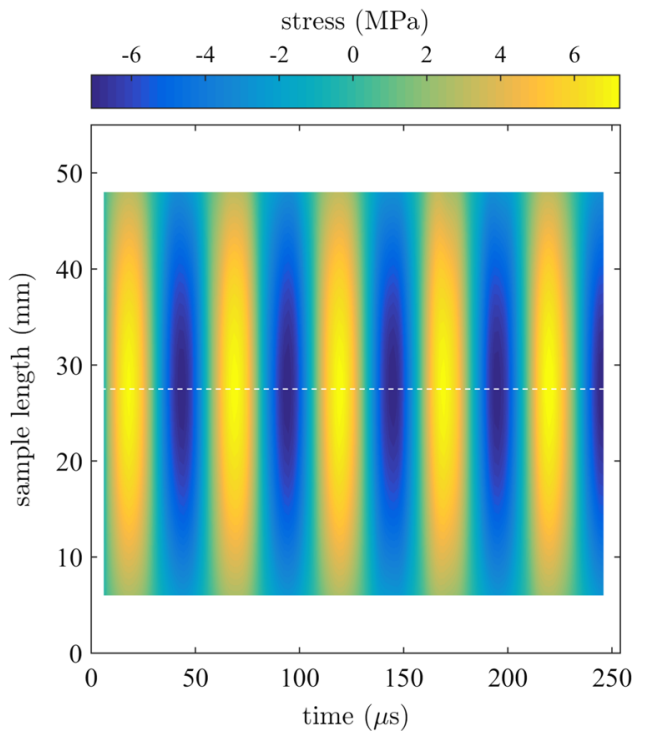

(d) stress temperature profile presented in Fig. 7(d) only results from a single frame. The strain and strain-rate data close to the free and fixed edges have been discarded because of edge artefacts from the Gaussian smoothing of the displacement fields. It is also important to note that the temperatures over $90{ }^{\circ} \mathrm{C}$ (see dashed rectangle in Fig. 7(d)) have been reconstructed using cubic spline interpolation. Indeed, due to the chosen integration time, the camera sensor saturated above $90{ }^{\circ} \mathrm{C}$. An integration time of about $400 \mu s$ or a multi-IT strategy would have been more suitable to capture the range [25 - 110] ${ }^{\circ} \mathrm{C}$.

One observes that, when the sample is loaded at \pm 735 $\mu$ def ( $1^{s t}$ series), the strain amplitude varies continuously along the sample length from 400 to $735 \mu$ def, the strainrate amplitude from 50 to $115 \mathrm{~s}^{-1}$, the stress amplitude from
0.2 to $4 \mathrm{MPa}$, and the temperature slightly increases in the sample centre from 23.3 to $34.6{ }^{\circ} \mathrm{C}$ while the edges remains at room temperature.

When the sample is loaded at $\pm 1542 \mu \operatorname{def}\left(2^{\text {nd }}\right.$ series), the strain amplitude varies from 200 to $1542 \mu$ def, the strain-rate amplitude from 40 to $220 \mathrm{~s}^{-1}$, the stress amplitude from 0.2 to $7.6 \mathrm{MPa}$, and the temperature increases in the sample centre from 23.4 to $105^{\circ} \mathrm{C}$. Contrary to the first test series where all curves overlapped almost perfectly, the second series evidences a softening of the material as demonstrated on Fig. 7(c). This is due to significant increase of the temperature cycle after cycle (see Fig. 7(d)). A clear change of the material response can be observed at the $4^{\text {th }}$ and $5^{\text {th }}$ ultrasonic runs. These two loading cases are characterized by a significant drop of the stress down to 6 
Fig. 7 Strain, strain-rate, stress and temperature profiles (along sample length) for each ultrasonic run. The free edge is at $0 \mathrm{~mm}$ while the fixed edge is at $55 \mathrm{~mm}$

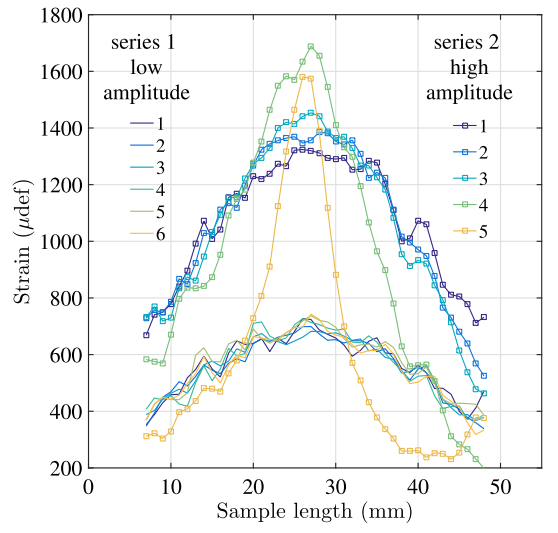

(a) strain

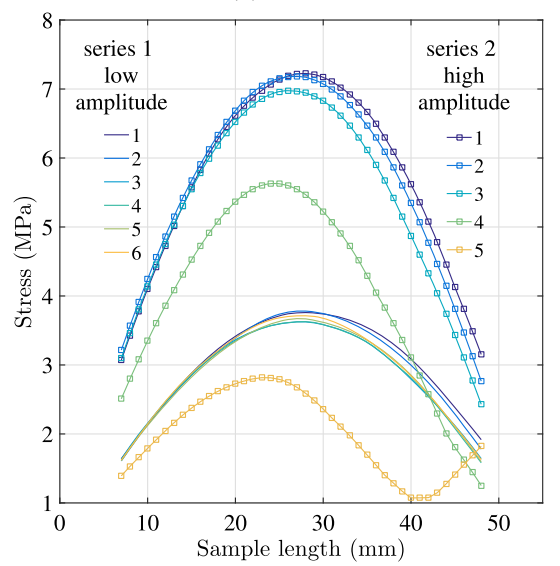

(c) stress

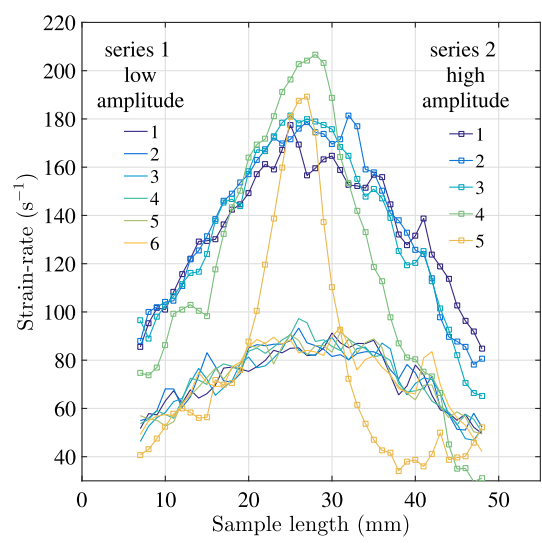

(b) strain-rate

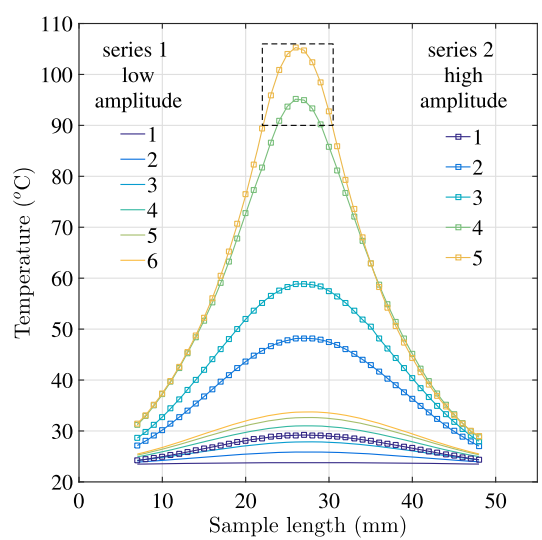

(d) temperature. Dashed rectangle underlines region where missing temperatures (due to IR sensor saturation) have been extrapolated. then $3 \mathrm{MPa}$ as well as clear change in the profile shapes. Indeed, one can see on Fig. 7(a) a clear strain localization in the sample centre and a significant decrease of the strain level everywhere else. These phenomena are due to a sharp localized change in the stiffness of PMMA due to glass transition. The phenomenon starts at the $4^{\text {th }}$ run, i.e. around $95^{\circ} \mathrm{C}$ but leads a clear collapse of the material stiffness at the $5^{\text {th }}$ run, i.e. around $105^{\circ} \mathrm{C}$. Such a drastic local change in material property also leads to a significant change in the deformation mode of the sample. Indeed, while the material is initially perfectly tuned to be resonant on its first longitudinal mode at $20 \mathrm{kHz}$, one observes a significant decrease of the wavelength (see Fig. 7(c)) at the $4^{\text {th }}$ and $5^{\text {th }}$ runs. This point is the reason why finite differences have been used to obtain experimental acceleration fields as the assumption of homogeneous material response cannot be ensured anymore. According to the wavelength formulation, available in "Theoretical Framework", a decrease of the wavelength relates to a drop of the stiffness which will be evidenced further. In the present case, the temporal resolution $(50 \mathrm{~Hz})$ of the temperature signal combined with the heating rate does not allow finely capturing the $\mathrm{T}_{g}$, but a value around $100{ }^{\circ} \mathrm{C}$ is reasonably in line with glass transition temperatures measured on the material using DMTA (see Table 1).

The data can now be combined to identify the material properties as a function of temperature and strain-rate.

\section{Storage Modulus and Damping Identification}

Figure 8 presents the evolution of the material response within three different sample cross-sections (at $\varepsilon= \pm 1542$ $\mu$ def, i.e. test series 2 - cf. Fig. 7) and for different ultrasonic runs. The dots represent the experimental data while the curves are based on a sinusoidal least-square fit. Both stress and strain are temporally fitted by a sine function as follows:

$\varepsilon_{f}(x, t)=\alpha_{0} \sin \left(2 \pi f t+\phi_{0}\right)$
$\sigma_{f}(x, t)=\alpha_{1} \sin \left(2 \pi f t+\phi_{1}\right)$

where $f$ is the loading frequency, $t$ is the time, $x$ is the axial position and $\alpha_{i}$ and $\phi_{i}$ are the amplitudes and phases of strain and stress for $i=0$ and $i=1$ respectively. From this 
Fig. 8 Stress-strain curves for 3 different sample sections, from free edge to sample centre, and for different ultrasonic runs during the $2^{\text {nd }}$ test series, i.e. high strain-rate amplitude

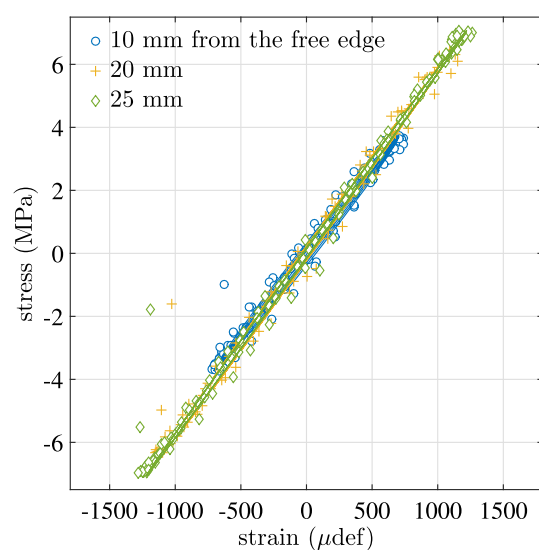

(a) run 1

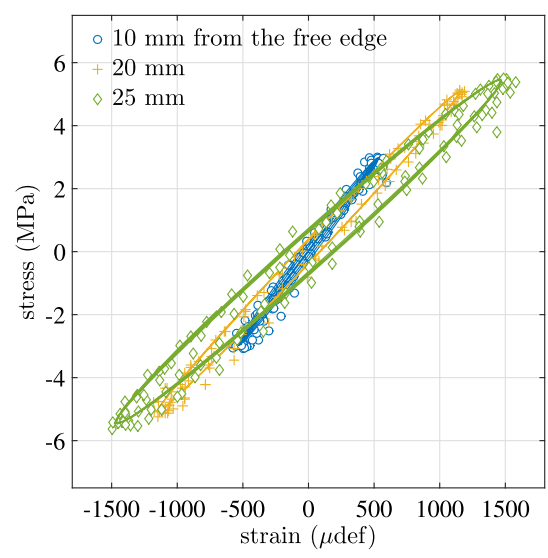

(c) run 4

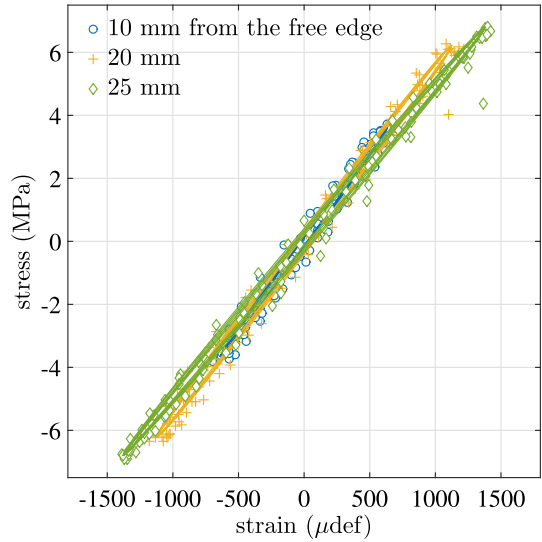

(b) run 3

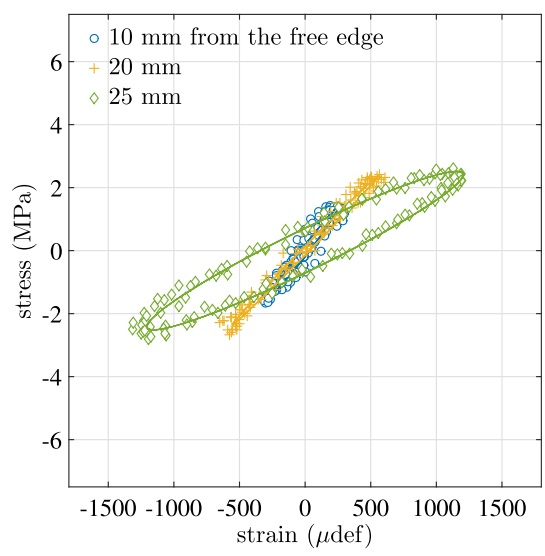

(d) run 5 description, $\tan (\delta)$ can also be obtained from the temporal phase shift between the stress and the strain as follows:

$\tan (\delta)(s, t)=\tan \left(\phi_{0}-\phi_{1}\right)$

This parameter is widely used to describe the damping of the material. The real part of Young's modulus, the storage modulus, is then identified from the ratio $\frac{\alpha_{1}}{\alpha_{0}} \cos (\delta)$.

The results show a number of important trends. Looking at Fig. 8(a) of the first run when the temperature in the specimen is still uniform (see Fig. 7(d)), one can see that the central sections provide a stiffer response than the outward one. This is the stiffening effect of strain-rate. Based on the data in Fig. 7(b), the material at $10 \mathrm{~mm}$ experiences a strain-rate of about $50 s^{-1}$ while the centre responds under a strain-rate of about $80 s^{-1}$. Both stiffness and strain-rate contrasts are not very large but the effect is significant. The second trend is the effect of temperature, which is much more prominent for this test. As the runs progress and the temperature increases at the centre of the sample, the stiffness drops as clearly seen in Fig. 8(c) and consistently over the whole test series. Finally, it is also clear from Fig. 8 that as the temperature increases, the hysteresis loops open up, indicating an increase in damping.
Based on the following local stress-strain relationships, the storage modulus and $\tan (\delta)$ have been identified and the results are presented in Fig. 9. Figure 9(a) presents the variation of storage modulus as a function of strain-rate and temperature. The red dots are the experimental data points obtained by combining information over 42 material sections (55 lines minus two filtering kernels to discard edges artefacts) and eleven ultrasonic runs, i.e. 462 data points. It is important to understand that each material slice is treated independently of its neighbours as if this was a separate material test. The continuous colour map is obtained by interpolating the data between the red dots using the Matlab ${ }^{\circledR}$ embedded natural neighbour interpolation method based on Voronoi tessellation. The figure shows the range of the current experiment, i.e. strain-rates between 30 and 220 $\mathrm{s}^{-1}$ and temperatures between about 25 and $105^{\circ} \mathrm{C}$. Within this $2 \mathrm{D}$ loading condition space, the storage modulus varies from $1.5 \mathrm{GPa}$, at high temperature, up to $6.3 \mathrm{GPa}$ at low temperature. The experiment clearly captures the temperature sensitivity of PMMA with a strong vertical gradient in the map in Fig. 9(a). Looking at the isothermal curves (horizontal gradient), a slight strain-rate sensitivity can also be noticed, as expected. This effect is small however as the strain-rate range covered here is rather small. One can also 
Fig. 9 Identification of storage modulus and $\tan (\delta)$ as a function of strain-rate and temperature.

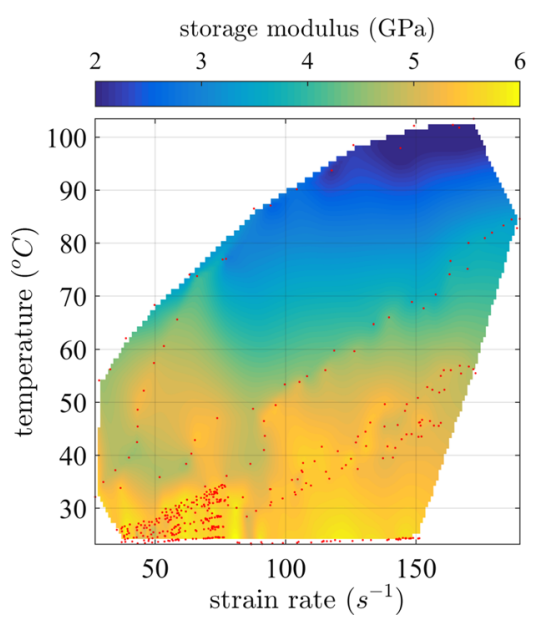

(a) stiffness

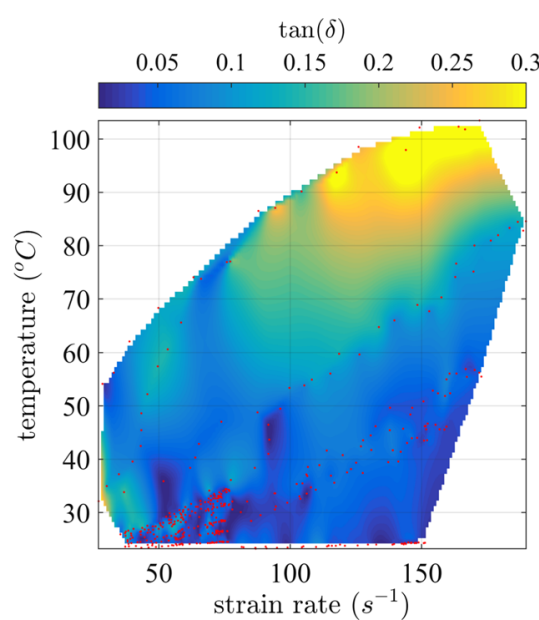

(b) $\tan (\delta)$ observe higher noise on the data for the lower strain-rate and temperature ranges. This corresponds to sections close to the specimen edges where both stress and strain are lower and therefore, exhibit degraded signal to noise ratios.

Then, Fig. 9(b) presents the variation of the $\tan (\delta)$ parameter which is associated to the material damping. A few excessively high and unrealistic damping values have been discarded (less than 10 data points higher than 0.4) and the colormap saturates at 0.3 to visually capture the local gradients. The same trend as for the storage modulus is observed, although the data are noisier. This was expected as $\tan (\delta)$ derives from small fractions of the total stress and strain. As for the storage modulus, the noise is larger in the low temperature and low strain-rate range, for the same reason. It is interesting to compare these data to that available in the literature. Looking for instance at [18], the variation of $\tan (\delta)$, from ambient to $105^{\circ} \mathrm{C}$, ranges between about 0.03 and 0.3 which is the range also observed here. Another interesting observation is that there is a trend for higher values at low strain-rate and temperature. This may be related to the presence of a $\beta$-transition around room temperature, but the poor signal to noise ratio there precludes any definite conclusion. More tests are needed to explore this part of the temperature / strain-rate space.

From a technical point of view, both graphs evidence a very heterogeneous data-point density. Indeed, Fig. 9(a) has a large significant data-point density between 50 and 100 $\mathrm{s}^{-1}$ for temperature comprised between ambient and $35^{\circ} \mathrm{C}$ whereas very few data-points are available at higher temperature. This point is not intrinsic to the method and mainly depends on the chosen ultrasonic loading amplitudes. In the present work, only two amplitude steps have been used $( \pm$ $735 \mu$ def and $\pm 1542 \mu$ def). Using more steps would have led to a more homogeneous space sampling.

Finally, time temperature superposition has been used in an attempt to collapse all the data in Fig. 9(a) on a single master curve. To achieve this, experimental data points undergoing low strain-rates $\left(<70 s^{-1}\right.$, see "Finite Element Validation" for details), and the ones close to $\mathrm{T}_{g}$ and post $\mathrm{T}_{g}$ have been discarded. For the latter, as the glass transition was not identified accurately, the procedure has consisted in removing data over a threshold damping value of 0.3 (see top yellow region in Fig. 9(b)) and a threshold temperature value of $95^{\circ} \mathrm{C}$.

For thermo-rheologically simple materials, i.e. materials that obey time-temperature superposition which is the case of PMMA below the $\mathrm{T}_{g}$, it is assumed that an increase in temperature is equivalent to a decrease in strain-rate. The reconstruction of a master curve consists therefore in shifting isothermal storage modulus curves along the strain-rate axis by a factor $a_{T}$ until a single curve is obtained, representing the evolution of the material behaviour at a fixed reference temperature $T$. The shifting factor $a_{T}$ can be described by the Arrhenius equation as follows:

$a_{T}(T)=\exp \left(\frac{\Delta H}{R}\left(\frac{1}{T}-\frac{1}{T_{0}}\right)\right)$

where $\Delta H$ is the activation energy associated with mechanisms of internal friction, $R=8.3144598 \mathrm{~J} \cdot \mathrm{mol}^{-1} \cdot \mathrm{K}^{-1}$ is the gas constant, and $T_{0}$ is a chosen reference temperature. Figure 10(a) shows the identified master curve at $25^{\circ} \mathrm{C}$. Storage modulus values are presented with scattered points (from blue (ambient) to red (hot)). Individual slopes of storage modulus isothermal curves are presented with multiple straight black lines and the master curve and its $\pm 5 \%$ and $\pm 10 \%$ vertical shifts are presented with plain and dotted lines respectively. In practice, to obtain such a master curve, storage modulus isothermal curves (horizontal slices in Fig. 9(a)) were first individually fitted by affine functions in order to obtain a first order description of their slopes. Such set of approximated isothermal curves can be 
Fig. 10 Master curves at $25^{\circ} \mathrm{C}$ on PMMA

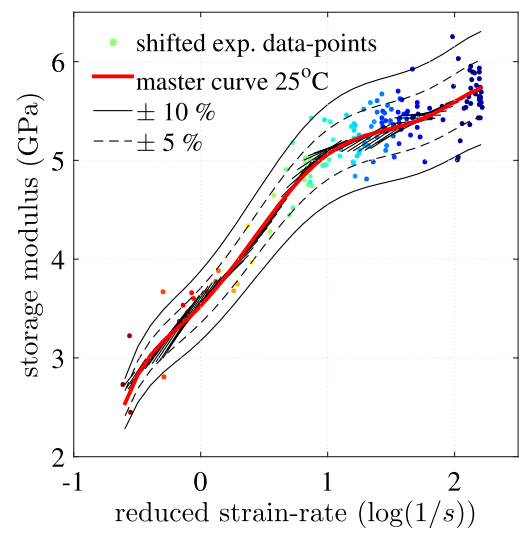

(a) Master curves reconstructed by horizontally shifting strain-rates according to Arrhenius law and based on an identified activation energy of $88 \mathrm{~kJ} \cdot \mathrm{mol}^{-1}$.

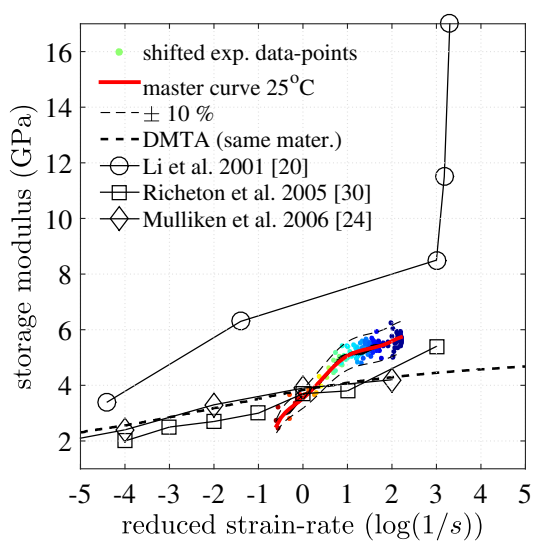

(b) Master curves with DMTA data obtained on same material are presented in dotted line as well as experimental data from Li et al. [20], Richeton et al. [30] and Mulliken et al. [24]. seen in Fig. 10(a) all along the master curve. Then, a $6^{\text {th }}$ order polynomial function was used to fit the whole set of approximated isotherms as a function of the reduced strainrate. Finally, a sweep on the activation energy value, thus on the shifting factor (see Equation (11)), was performed and the difference between the isotherms and the polynomial approximation calculated. An identified activation energy of $88 \mathrm{~kJ} \cdot \mathrm{mol}^{-1}$ has been found to minimize this deviation in the least-squares sense. The identified activation energy is in same order of magnitude than values found in the literature, see e.g. [1] where an activation energy of $71 \mathrm{~kJ} \cdot \mathrm{mol}^{-1}$ has been found for PMMA samples tested with a Broadband Viscoelasticity Spectrometer (BVS), or $90 \mathrm{~kJ}^{\mathrm{mol}}{ }^{-1}$ in [11]. In addition, it is worth mentioning that the scatter of the storage modulus falls within a $\pm 10 \%$ error band around the master curve, with a decrease of the scatter down to $\pm 5 \%$ at higher temperatures, and that the isotherms follow reasonably well the trend over 2 decades of strain-rate. Such a comparison demonstrates the relevance of the data extracted from the current ultrasonic experiment.

The reconstructed master curve at $25^{\circ} \mathrm{C}$ is also presented in Fig. 10(b) with a set of data from the literature. In addition, DMTA was performed on the same material using a Q800 system from TA Instruments. A stepwise temperature control with a $1{ }^{\circ} \mathrm{C}$ interval between each step was used during the measurements. The testing temperature ranged from $-80^{\circ} \mathrm{C}$ up to $50^{\circ} \mathrm{C}$ and the data were recorded at multiple frequencies: $0.1,0.3,1,3,10$ and $30 \mathrm{~Hz}$. The 17.25 $\times 12.07 \times 3 \mathrm{~mm}^{3}$ specimen was subjected to a dual cantilever deformation mode ${ }^{4}$ and the strain amplitude was kept lower than $0.1 \%$. Comparing the present "one-shot" material characterisation to DMTA and literature results, a certain discrepancy can be observed (see Fig. 10(b)). The present results are located between that from [20], where the

\footnotetext{
${ }^{4}$ Similar to 3 -point bending except that ends of the sample are clamped.
}

material was subjected to compressive tests at quasi-static (servo-hydraulic machines) and high strain-rates (SHPB), and $[24,30]$ with data from DMTA and compressive tests on both servo-hydraulic machines and SHPB. The trends are similar but the current data lie about $1 \mathrm{GPa}$ above that from $[24,30]$, while they are about $2 \mathrm{GPa}$ below that in [20]. An interesting thing is the fact that the data in [30] and [20] as well as the current data (partially) evidence an inflection in the behaviour somewhere between $10^{1}$ and $10^{3} \mathrm{~s}^{-1}$, which is not visible on both DMTA result. Nevertheless, nore experiments, at higher strain-rates, or at lower temperatures (through the time-temperature superposition principle) are be necessary to confirm the existence of such a sharp increase of the material stiffness at high strain-rate as well as the ability of high-speed tests, contrary to standard DMA, to capture it.

The focus of the present work is not to perform a thorough study of the viscoelasticity of PMMA but to propose a truly new test method to reach strain-rates that are generally hard to obtain as they lie between what can be obtained with high speed hydraulic machines on one side and split Hopkinson bars on the other side. The data in Fig. 10(b) shows the benefit of having an alternative to the current test techniques to better explore the behaviour of viscoelastic materials at high rates of strain directly, without having to rely on time-temperature superposition, as for DMTA tests. The lack of transition at high strain on the DMTA data is a hint that this test may be missing some important transition. The difficulties associated with tests on high speed hydraulic machines and SHPB contrast with the relative simplicity of the current configuration and the present authors strongly believe that this new test can be a valuable addition to the toolkit of the mechanics of materials researchers and engineers.

The objective of the last section is to shed some light on the issue of the low spatial resolution of the camera. Indeed, the digitized measurements provided by the camera and the 
grid method are a spatially filtered version of reality and the only way to understand the effect of this filter on the quality of the identification is through numerical simulation.

\section{Finite Element Validation}

The purpose of this section is to validate the current experimental choices by understanding how experimental parameters such as the camera spatial resolution, the acquisition frequency, the grid sampling, the camera sensor noise and the sensitivity of the algorithm used to recover deformations can affect the precision of the identification. The idea is also to propose some guidelines to the reader and make this new technique more accessible. The main concern is the poor camera spatial resolution $(400 \times 250$ pixels $)$ so this validation is essential to give confidence to the previous results.

\section{Finite Element Model}

The Finite Element (FE) simulation consisted in an 3D harmonic analysis achieved using the ANSYS 16.2 FE package. Thanks to the problem symmetry, only a quarter of the sample was modelled. The model was meshed using 0.1 $\mathrm{mm}^{3}$ SOLID186 quadratic elements and a harmonic loading of amplitude $30 \mu \mathrm{m}$ was applied to the right-hand side edge of the model (see Fig. 11).

The material was modelled using an isotropic, homogeneous and purely elastic material. In other words, the present FE investigation does not deal with the impact of material time-dependence, i.e. impact of temperature and strain-rate variations along the sample length, nor the dissipation, on the identification validation process. This was found reasonable as a first step to quantify the effect of the camera spatial resolution on the measured and identified quantities. The main characteristics of the FE model are summarized in Table 2.

Figure 11 shows an example of an FE longitudinal displacement field extracted at the top surface of the model and then symmetrized along the transversal direction.

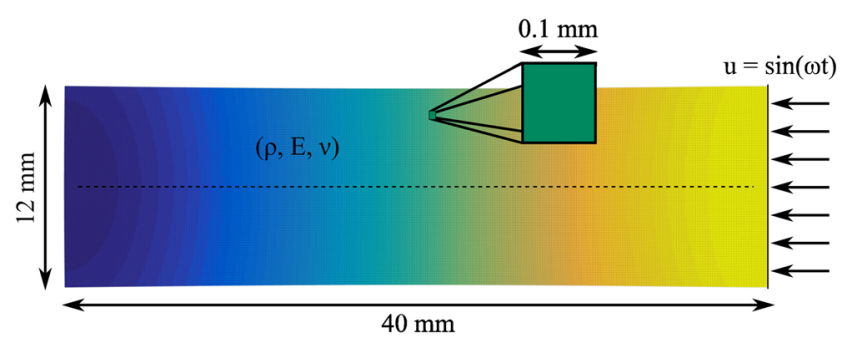

Fig. 11 Example of FE displacement field extracted at the top surface of the FE model and symmetrized along the transverse direction

\section{Synthetic Grid Deformation}

The procedure used to evaluate the accuracy of the identification on real data is presented here. First, the sample deformation was simulated with the FE model described above. Then, synthetic grid images were numerically deformed using the FE displacements fields, grey level noise was added and the images were processed exactly as the experimental ones (see "Theoretical Framework"). The identified parameters can then be compared to the FE inputs and both systematic and random errors can be evaluated. A similar approach can be found in [32, 33, 38].

Synthetic and pixelated grids have been generated according to the following equation:

$G(i, j)=\frac{\gamma}{4}\left(1+\cos \left(2 \pi \frac{i}{N}\right)\right)\left(1+\cos \left(2 \pi \frac{j}{N}\right)\right)+\tilde{\gamma}$

with $G$ is the pixelated grid, $N$ is the spatial sampling (number of pixels per period) and $\gamma$ and $\tilde{\gamma}$ characterises the image dynamic range (see Fig. 12). A specific grid pitch $p$ was also chosen to scale the image. In parallel, FE displacement fields were output from ANSYS and interpolated on the grid-mesh using a cubic spline interpolation. Such an interpolation is required to deform the grid using standard MATLAB libraries such as the interp 2 function. Finally, the grid was numerically deformed, assigning at grid location $M(i, j)$ an average of the grid grey level value around the location $M^{\prime}(i+u, j+v)$ where $u$ and $v$ are the longitudinal and transversal FE displacement at that pixel location, respectively. Additional spline interpolations were required here to estimate the grey level value at locations $M^{\prime}(i+u, j+v)$ and $M^{\prime}(i+u \pm \epsilon, j+v \pm \epsilon)$, where $\epsilon$ is half the averaging window size. It corresponds to the field of view captured by one pixel of the camera, denoted pix, and allows taking into account the fact that a camera pixel only captures an average of the grid grey level over a certain physical domain. Here, this window was taken so that it covers the full pixel size, effectively simulating a fill factor of $100 \%$, while the Shimadzu HPV-X camera has a low fill factor, of the order of $40 \%$. Nevertheless, it was checked numerically that this effect could be neglected thanks to the very small recorded displacements (less than a third of a pixel), justifying the use of a $100 \%$ fill factor here for the sake of simplicity and computing efficiency. Finally, a grey level Gaussian noise of standard deviation $\sigma$ was added to the grid image to take into account the characteristic noise of the camera sensor. Such routine is applied to the initial grid image for different loading increments until reconstructing 127 synthetically deformed and noisy grid images comparable to the data set captured by the camera during a real experiment. 
Table 2 FE model parameters

\begin{tabular}{lll}
\hline Geometry & dimensions $(\mathrm{mm})$ & $55 \times 6$ (sym.) x 2 (sym.) \\
Mesh & size $\left(\mathrm{mm}^{3}\right)$ & 0.1 \\
& element & SOLID 186 \\
Material & density $\left({\left.\mathrm{kg} . \mathrm{m}^{-3}\right)}\right.$ & 1160 \\
& Young's modulus $(\mathrm{GPa})$ & 5.5 \\
& Poisson's ratio & 0.34 \\
Loading & type & disp. \\
& amplitude $(\mu \mathrm{m})$ & 30 \\
& frequency $(\mathrm{kHz})$ & 20 \\
Analysis & FE package & ANSYS 16.2 \\
& type & harmonic / full \\
& solver & Jacobi Conjugate Gradient itera- \\
& & tive equation solver \\
& num. damping $(\mathrm{s})$ & $\times 10^{-7}$ \\
\hline
\end{tabular}

Figure 12(c) shows a zoomed in image of an experimental grid image and Figs. 12(a) and (b) the grey level histogram and noise characteristics of the camera sensor. Figure 12(c) shows a zoom in of the synthetic grid after applying deformation and noise. The noise added to the synthetic images is based on Fig. 12(b) where the standard deviation of the pixel noise (obtained from stationary images) for different pixels from dark to bright is plotted.
The maximum noise was about $3.5 \%$ of the pixel grey level and decreases as a function of the grey level intensity. The grid contrast has been selected to reproduce the histogram of Fig. 12(a). Comparing Fig. 12(c) and (d), one can note that the procedure detailed above describes reasonably well the grey level dynamic and the noise intensity. However, the procedure does not take into account any grid defect and any fill-factor issues. Indeed, one observes in Fig. 12(c) that the
Fig. 12 Experimental images characteristics a), b) and zoom in views of an experimental and synthetic grid. The corresponding parameters are: $p$ $=1 \mathrm{~mm}, N=7 \mathrm{~mm}$, pix $=143$ $\mu m$ and $\sigma=3.5 \%$
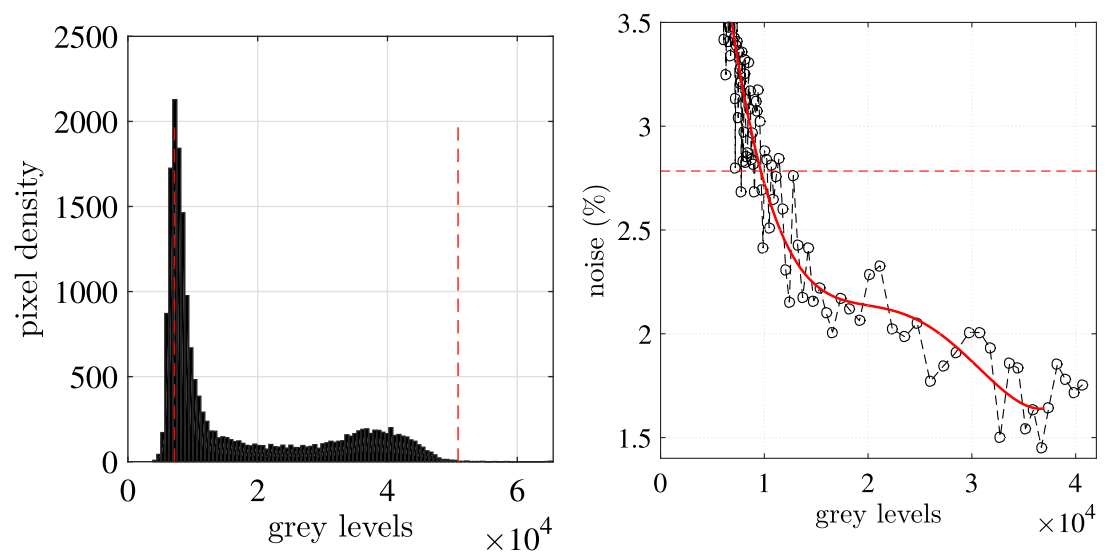

(a) Experimental grid histogram

(b) Experimental grid image noise as a function of pixel grey levels

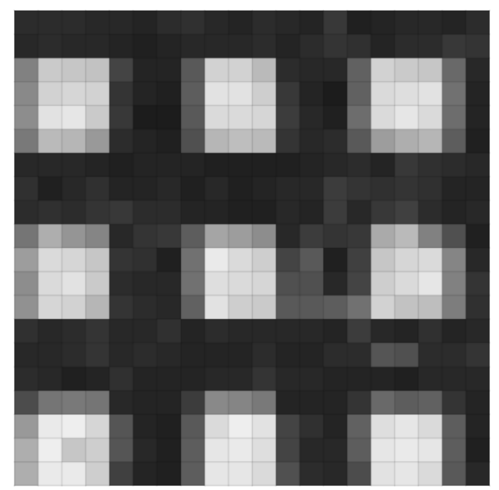

(c) Experimental grid image

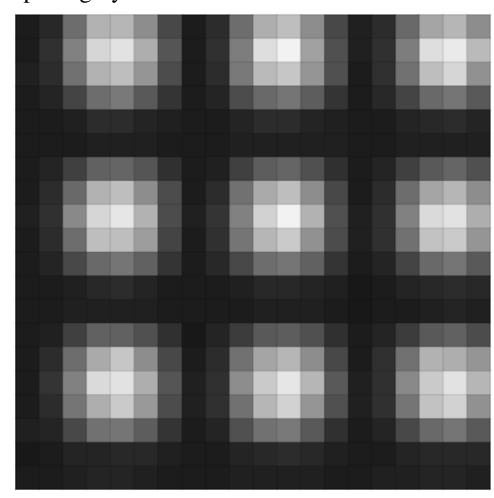

(d) Synthetically deformed and noised grid 
Fig. 13 Spatio-temporal displacement error maps. On the left column is the absolute value of the relative error and on the right column is the absolute value of the extracted random part. The corresponding parameters are: $p=1 \mathrm{~mm}, N=$ $7 \mathrm{~mm}$, pix $=143 \mu \mathrm{m}, \sigma=3.5$

$\%$ and smoothing kernel $s=7$

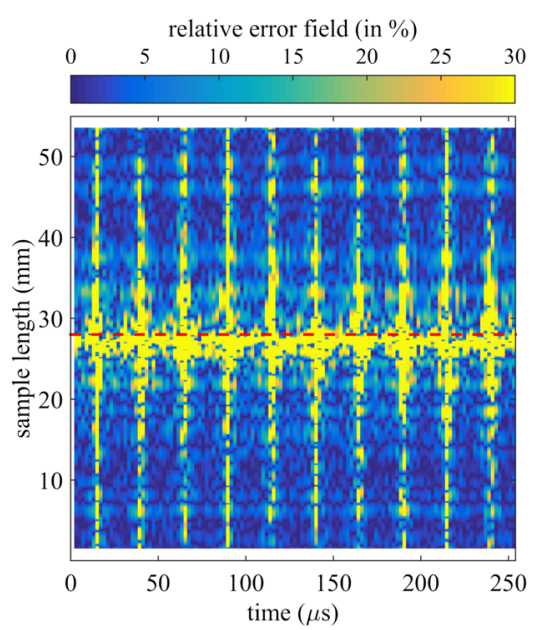

(a) Relative error on raw displacement: $\varepsilon^{\text {syst }}=0.2 \%$

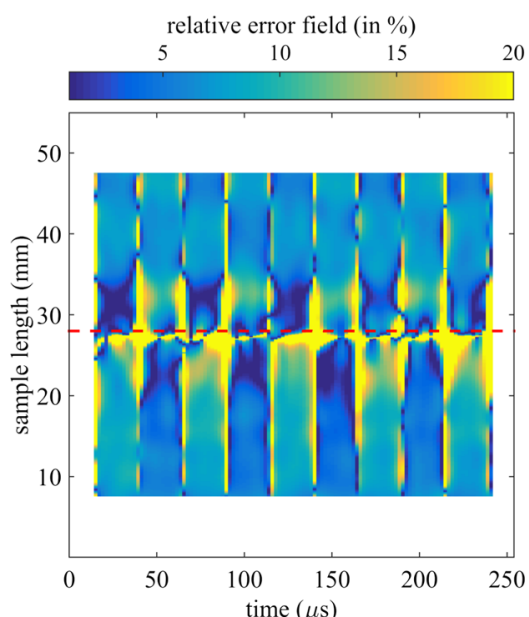

(c) Relative error on smoothed displacement: $\varepsilon^{\text {syst }}=6.7 \%$

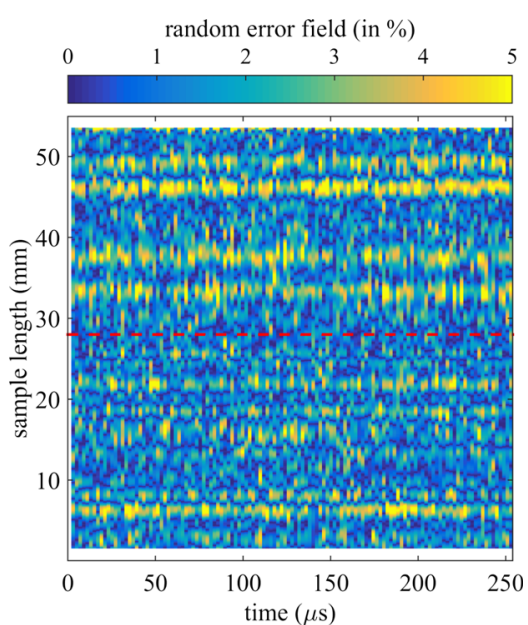

(b) Extracted random error part: $\varepsilon^{\text {rand }}=1.7 \%$

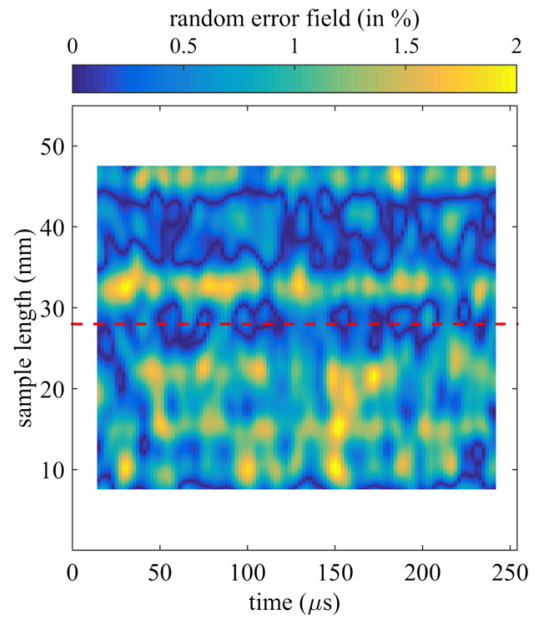

(d) Extracted random error part: $\varepsilon^{\text {rand }}=0.6 \%$ apparent pitch of the grid slightly varies along the horizontal direction. This is due to the fact that the pixel captures photons only over a small part of its physical domain ( $40 \%$ for the HPV-X) which leads to a crop of the information especially visible in presence of sharp edges. Both these aspects have been neglected here.

Before analysing the impact of the grid and camera parameters on the field reconstructions, one needs to choose a definition for the systematic and random errors, i.e. scalars accurately describing spatio-temporal deviations between input and output fields. The idea was to overcome two major problems: (1) the presence of infinite values in the definition of the relative error. It comes from the point-by-point division of the output fields by the input ones, of which the local values can be null. (2) By smoothing the reconstructed displacement field, the random noise will be mitigated but outputs are more and more truncated which introduces low spatio-temporal frequency variations within the error. This makes it difficult to simply define the random error by the standard deviation of the relative error. The following two points are illustrated in Fig. 13 where the relative error on displacement fields (see Equation (13)) and its random part (see Equation (14)) are presented for a simulated camera noise of $3.5 \%$ with smoothing (Fig. 13(c) and (d)) or without (Fig. 13(a) and (b)). As expected, singular values close to the anti-node (sample centre) are present as well as for certain times when the signals go through zero. One can see the reduction of high frequency scatter when smoothing (from top to bottom) but also the creation of systematic low-spatial frequency variations (see Fig. 13(c)). Based on these observations, it has been decided to define the systematic error, denoted $\epsilon^{s y s t}$, as the median (over space and time) of the relative error field as the median is less sensitive to outliers arising from low signal points. The random error, denoted $\epsilon^{\text {rand }}$, is defined as the standard deviation (over space and time) of the difference between the FE data 
corrected by the systematic error and the FE-grid data, normalized by the maximum of the signal in space and time. In equation format:

$$
\begin{aligned}
\epsilon^{\text {syst }} & =M_{d}\left[\frac{F^{F E}(x, t)-F^{\text {grid }}(x, t)}{F^{F E}(x, t)}\right] \\
\epsilon^{\text {rand }} & =s\left[\frac{F^{F E}(x, t)\left(1-\epsilon^{s y s t}\right)-F^{g r i d}(x, t)}{\max _{x, t}\left(F^{F E}(x, t)\right)}\right]
\end{aligned}
$$

where $F^{F E}$ and $F^{\text {grid }}$ are the FE (input) and post-processed (output) quantities of interest, $M_{d}[*]$ and $s[*]$ are the median and the standard deviation respectively. For the error on Young's modulus, the time has been removed from Equations (13) and (14) to calculate the error, as it does not depend on time.

Let us now look into the impact of the experimental spatial grid sampling, the temporal sampling and the camera sensor noise on measurements and identification. First, four different spatial grid pitches have been tested, namely $p=$ $0.7,0.8,0.9$ and $1 \mathrm{~mm}$, and the error on kinematic quantities evaluated. No clear impact on the systematic error of displacement and strain has been identified since an error of $0.5^{ \pm 0.1} \%$ on both displacement and strain has been found whatever the grid pitch. Therefore, the grid pitch chosen experimentally, $1 \mathrm{~mm}$, has negligible impact on the displacement and strain. The reason for this is certainly the low spatial frequency contents of the deformation when the material undergoes deformation at its first longitudinal mode. Deforming the material at higher deformation modes, or using a strain concentrator like a notch or a hole, would require to run this check again.

Then, five different frame rates have been used, namely $f=0.2,0.5,1,2$ and $5 \mathrm{Mfps}$, on a $1 \mathrm{~mm}$ pitch grid. Table 3 summarizes the results.

One observes that only the $0.2 \mathrm{Mfps}$ frame rate significantly affects the results. Indeed, an error of about $12 \%$ is found for simulation recorded at $0.2 \mathrm{Mfps}$, while only 1-2 \% of error was found for higher frame rates. This confirms the choice of $0.5 \mathrm{Mfps}$ for the experiments, and also

Table 3 Systematic error (in \%) between FE and synthetic grid deformation data, for different frame rates. The grid pitch is $1 \mathrm{~mm}$ and the displacement amplitude is $\pm 30 \mu \mathrm{m}$

\begin{tabular}{lcccccc}
\hline \multicolumn{5}{c}{ Frame rate (Mfps) } \\
\cline { 2 - 6 } & 0.2 & 0.5 & 1 & 2 & 5 \\
\hline Accel. (finite diff.) & 11.6 & 0.9 & 0.7 & 1.1 & 1.2 \\
Accel. (harm.) & $0.3 \rightarrow$ & & & \\
Stress (finite diff.) & 11.4 & 0.7 & 1.0 & 1.7 & 1.9 \\
Stress (harm.) & $0.14 \rightarrow$ & & & \\
Young's modulus (finite diff.) & 12.3 & 1.9 & 0.3 & 0.5 & 1.2 \\
Young's modulus (harm.) & $0.5 \rightarrow$ & & & \\
\hline
\end{tabular}

shows that a slight improvement would have been obtained recording at $1 \mathrm{Mfps}$ or more. It is interesting to notice that the small unexpected increase of the identification error over $1 \mathrm{Mfps}$ is probably due to the fact that the systematic errors presented here are based on spatio-temporal data obtained considering a constant number of frames captured by the camera (128) and not considering a constant number of deformation cycles. This has been chosen to be in line with the physical limitation of the camera. In other words, when an increase of the acquisition frequency is simulated, a decrease of the number of captured deformation cycles occurs. At $1 \mathrm{Mfps}$, two and a half cycles are captured, at 2 Mfps only one and at $5 \mathrm{Mfps}$, only half of a cycle. Therefore, increasing the frame rate affects the consistency of the comparison and probably slightly increases the systematic error. However, this error still remains very small. The table also shows that avoiding temporal differentiation by using the harmonic assumption leads to lower errors on the acceleration and stress, as expected.

Finally, a noise level of $3.5 \%$ was added to the synthetically deformed grid images, each of the 128 images bearing a different copy of the noise. Table 4 and Fig. 14 present how displacement smoothing affects the strain, acceleration, stress and identified Young's modulus. For comparison, Fig. 13 provide the error fields corresponding to the first row, column one and two in Table 4. The first column of Table 4 shows the level of noise before any post-smoothing. No systematic error is observed but a significant random error can be seen on differentiated quantities, especially the strain and the acceleration with a noise standard deviation of about $11 \%$ and $13 \%$, respectively. One can see that the random error level is only about $6 \%$ on stress (finite diff.). This point is in line with the fact that the stress comes from averaging the acceleration between each material section and the free edge. This is a regularizing process. In addition, one observes that the harmonic assumption

Table 4 Systematic and random errors (in \%) between FE and synthetic grid deformation data.

\begin{tabular}{lll}
\hline & no smoothing & smoothed k=7 \\
\hline Displacement & $0.2^{ \pm 1.7}$ & $6.7^{ \pm 0.6}$ \\
Strain & $0.4^{ \pm 11.0}$ & $6.7^{ \pm 2.7}$ \\
Acc. (finite diff.) & $0.6^{ \pm 12.9}$ & $7.2^{ \pm 1.5}$ \\
Acc. (harm.) & $0.2^{ \pm 1.7}$ & $6.7^{ \pm 0.6}$ \\
Stress (finite diff.) & $0.1^{ \pm 5.7}$ & $7.0^{ \pm 1.2}$ \\
Stress (harm.) & $0.2^{ \pm 1.0}$ & $6.6^{ \pm 0.8}$ \\
Young's modulus (finite diff.) & $2.8^{ \pm 3.1}$ & $1.4^{ \pm 1.8}$ \\
Young's modulus (harm.) & $1.5^{ \pm 3.2}$ & $0.8^{ \pm 1.8}$ \\
\hline
\end{tabular}

The image noise was $3.5 \%$ of the grey level value and the displacement amplitude $\pm 30 \mu \mathrm{m}$. Smoothing was Gaussian with kernel $k$, in space for strains, and in time for acceleration (finite difference). Data provided as systematic ${ }^{ \pm \text {random }}$ error 


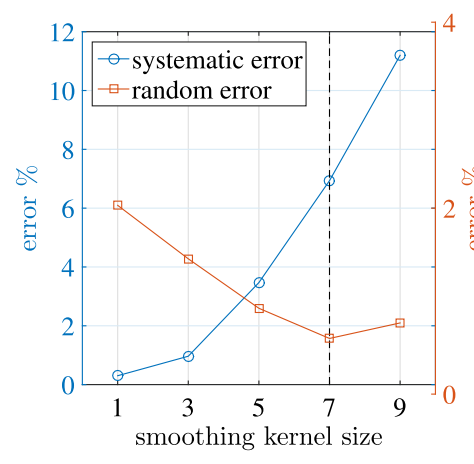

(a) Displacement

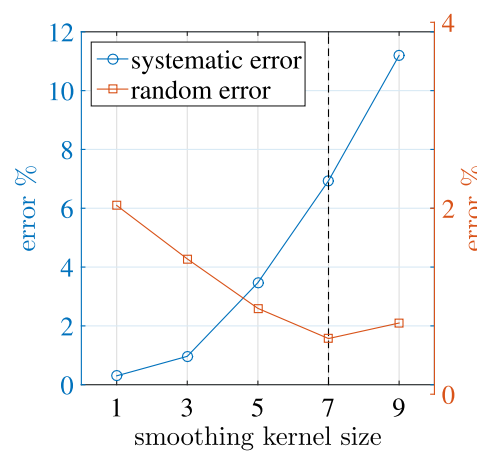

(d) Acceleration (harm.)

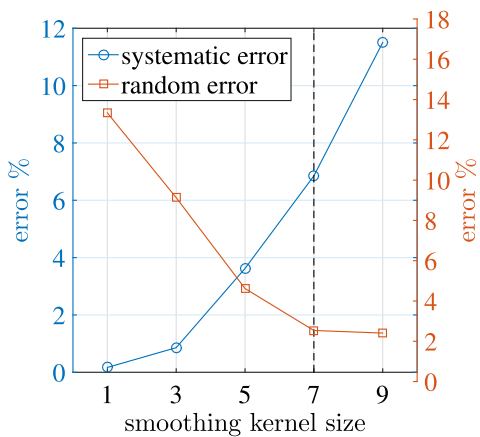

(b) Strain

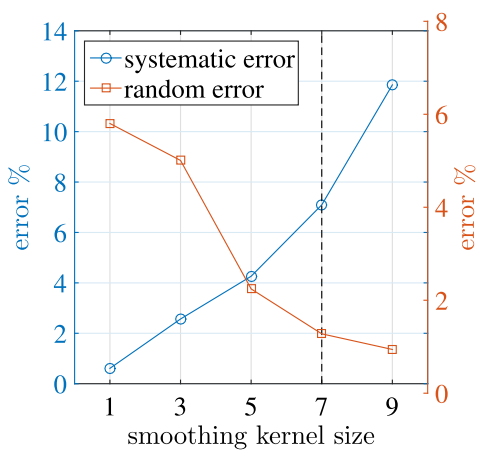

(e) Stress (finite diff.)

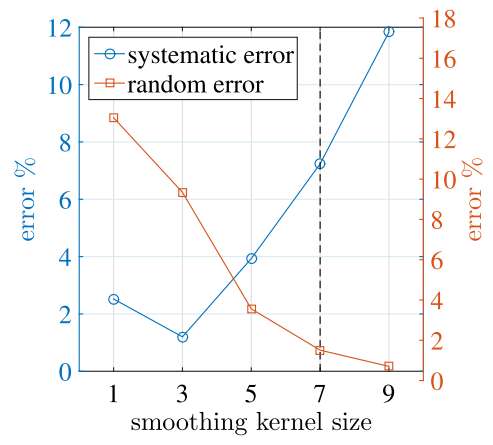

(c) Acceleration (finite diff.)

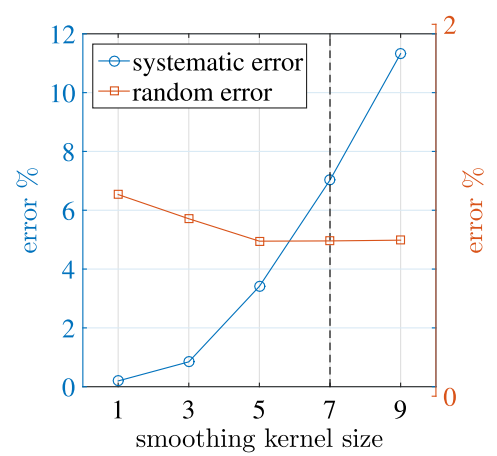

(f) Stress (harm.)

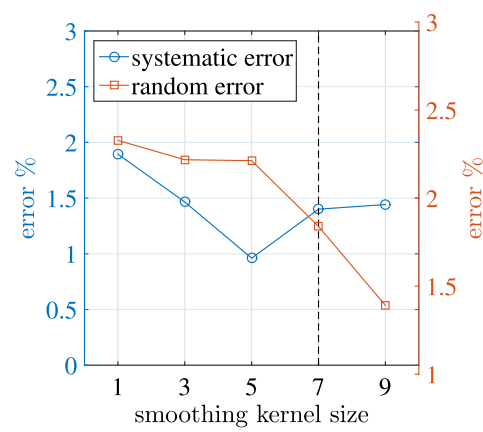

(g) Young's modulus (finite diff.)

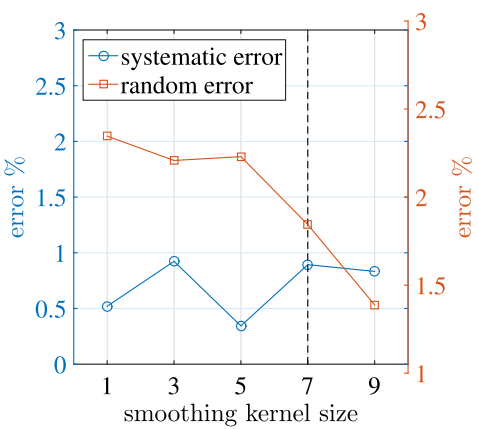

(h) Young's modulus (harm.)

Fig. 14 Systematic and random errors (in \%) between FE and synthetic grid deformation data. The image noise was $3.5 \%$ of the grey level value and the displacement amplitude $\pm 30 \mu \mathrm{m}$

significantly mitigates the influence of the noise. Both harmonic acceleration and stress have a level of noise similar to the displacement one. Finally, one can see that the identified Young's modulus has a systematic error which is about twice as large when using finite differences instead of the harmonic assumption to derive the acceleration. The latter is therefore favoured, as expected.

Table 4, column two, focuses on the smoothing kernel size selected for the treatment of the experimental data. A kernel size of 7 was applied in space to derive the strains, and in time to derive the acceleration using finite differences. Two things can be noticed: first, smoothing the data introduces a systematic error of the order of $7 \%$ for all quantities except the identified Young's modulus. Second, it significantly reduces the random error for the differentiated quantities, i.e. the strain (-8\%), the acceleration (-11\%) and the stress $(-4.5 \%)$. As expected, the random error does not reduce significantly on acceleration and stress when using the harmonic assumption, the smoothing just increasing the systematic error. The situation for Young's modulus is somewhat unexpected as not only does the random error decrease, as expected, but also the systematic error by a factor of two. This comes from the fact that both stress and strain systematic errors cancel out when the modulus is calculated. Whether this is a general result or just a fortunate fact arising from the precise set of smoothing parameters used here remains to be confirmed.

Finally, Fig. 14 shows the variation of the systematic and random errors when the smoothing kernel size varies. For all quantities, the systematic error increases when the 
smoothing kernel increases and the random error decreases when the kernel increases, as expected. This allows to select the smoothing in a rational way to minimize the total error, as in [38]. It is clear from Fig. 14(b), (c) and (e) that a smoothing kernel between 5 and 7 is recommended. Regarding the identified Young's modulus, it is interesting to notice that the trend of the systematic error is almost flat whereas the systematic error on both strain and stress increases as a function of smoothing kernel size. It means that the systematic errors on both stress and strain increase in similar ways and cancel out in the Young's modulus identification. This specific point means that depending on the purpose of the study, i.e. identifying a material parameter or measuring accurately mechanical quantities, the choice of the smoothing kernel will not necessarily be the same. In parallel, one observes that the random error on Young's modulus identification significantly decreases over a smoothing window of 5 . Nevertheless, none of the tested parameters allow reaching an error level below $1 \%$. A wider range of smoothing and grid parameters would be needed to better understand this, but this was beyond the scope of the present validation which focuses on evaluating the expected error for the parameters used in the experimental study. These results also demonstrate the benefit of the synthetic image deformation process to gain insight into the errors than can be expected on the identified quantities, as already pointed out in $[32,33,38]$.

To conclude, Fig. 15 presents the relative error expected on Young's modulus identification for the experimental conditions presented in "Results and Discussion", i.e. a grid pitch of $1 \mathrm{~mm}$, a frame rate of $0.5 \mathrm{Mfps}$, a flat camera sensor noise across pixel grey level values of $3.5 \%$ and a data post-smoothing kernel of 7 prior to both spatial and temporal differentiation. In view of the simplifications in the FE model, in particular the fact the material does not include any viscoelastic effects, i.e. no Young's modulus variations along the sample length are present, the results can be seen as a lower bound of the identification error. Figure 15(a) presents the relative error on the identified Young's modulus all along the sample length. The dotted red line represents the median value already reported in Table 4 . One observes a $3 \%$ oscillation of the identified Young's modulus along the sample length, from 10 to $40 \mathrm{~mm}$. Between 5 and $10 \mathrm{~mm}$, and 40 and $50 \mathrm{~mm}$, oscillations reach $10 \%$. Close to the free $(0 \mathrm{~mm})$ and the loaded $(55 \mathrm{~mm})$ edges, the identification error shoots up. This is due to the fact that both stresses and strains are zero at the deformation nodes. It is worth noting that the sample extremities (one smoothing kernel, i.e. 7 data, at both ends) have not been taken into account for the estimation of the systematic and random errors presented within this section. Such a figure shows that the accuracy of the identification is not constant over the sample length and depends on the position of the material section compared to the deformation nodes and anti-nodes. Indeed, Fig. 15 presents a characteristic $U$ shape which derives from the spatial variation of the signal to noise ratio. Here, the spatial location can be translated into strain-rate in order to underline the resulting impact of such signal to noise ratio variation on the ability to the experiment to characterize mechanical properties at lower strain-rate.

Figure 15(b) presents the relative error on the identified Young's modulus as a function of the strain-rate amplitude seen by each sample section. One clearly sees that the relative error on Young's modulus starts from $1 \%$ at maximum strain-rate (i.e. sample centre) then gradually increases as the considered section gets closer to the edges. Within the domain [200-250] s ${ }^{-1}$, the systematic error remains below $2 \%$ and reaches $6 \%$ down to $90 \mathrm{~s}^{-1}$. Below this, Young's modulus is not identified accurately anymore. Such observations are in line with the experimental observations. Indeed, Fig. 10(a) shows an identification scatter about \pm 5 $\%$ at the sample centre (high temperature - high strain-rate) and about $\pm 10 \%$ close to sample edge which is consistent

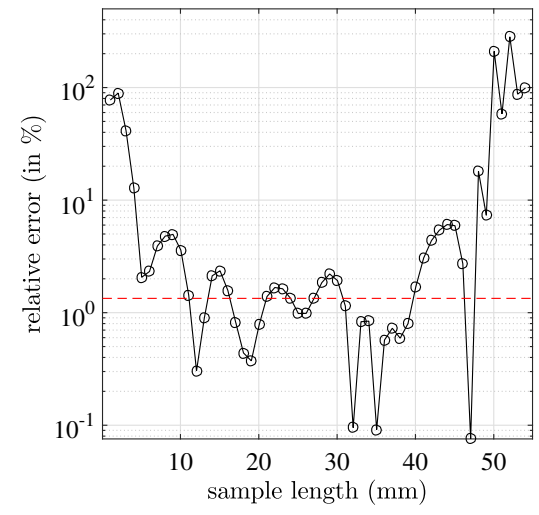

(a) Error as a function of sample length

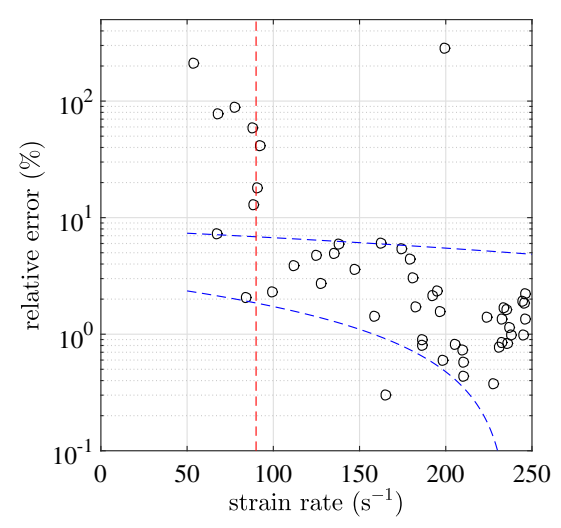

(b) Error as a function of the local amplitude of strain-rate

Fig. 15 Absolute value of the relative error on Young's modulus identification for a grid pitch of $1 \mathrm{~mm}$, a temporal sampling $\mathrm{f}=0.5 \mathrm{Mfps}$, a grid noise of $3.5 \%$ and a spatio-temporal smoothing kernel size of $7^{2}$. The material was assumed, isotropic, homogeneous and purely elastic. The displacement amplitude is $\pm 30 \mu \mathrm{m}$ and the acceleration was obtained from finite difference. The red line in a) is the median value while the red line in b) underlines the limit $\left(90 \mathrm{~s}^{-1}\right)$ under which the identification error shoots up 
with Fig. 15(b). Moreover, Fig. 15(b) shows that the systematic error slightly increases close to the sample edges which could partially explain the deviation with [30], observed in Fig. 10(a) at $10^{2} \mathrm{~s}^{-1}$.

The present data lead to the following conclusions. (1) Combining a spatially heterogeneous loading together with kinematic and temperature full-field measurements, it is possible to identify Young's modulus over a certain range of thermo-mechanical loading conditions. (2) The accuracy of the local identification strongly depends on experimental conditions and processing parameter selection, especially the sensor noise, the acquisition frequency, the camera resolution, the grid resolution and the smoothing parameters. Such parameters can be chosen in a rational way by combining finite element modelling and synthetic grid deformation. (3) Nevertheless, with such a "one-shot" technique, the cost to pay is a variation of the signal to noise ratio as a function of spatial location, i.e. also as a function of temperature, strain and strain-rate. Here, the results are acceptable down to strain-rates of $90 \mathrm{~s}^{-1}$ and up to $250 \mathrm{~s}^{-1}$. Deeper investigations using thermo-mechanical viscoelastic simulations are required to gain better understanding of the metrological limitations of the experiment.

\section{Outstanding Issues and Scope of the Method}

Although the feasibility of a "one-shot" time-dependent properties identification has been demonstrated on a PMMA sample and the results are in good agreement with data obtained from other methods, such as DMTA and highspeed compressive tests, it is important to understand the limitations and outstanding issues of the proposed methodology. A few points need to be raised.

- The difficulty to clearly define an apparent strainrate. Such difficulty is actually also present in DMTA and SPHB tests but at least the methodologies are consistent since data are also averaged in space. In the present case, the following issue can introduce a horizontal shift of the data in Fig. 9. As the strain-rate for result reported here is the maximum strain-rate, effective properties may be slightly shifted towards lower strain-rates. One can notice that the convention in DMTA is to approximate the strain-rate by $\dot{\varepsilon}=4 f \varepsilon_{\max }$ [24] whereas one uses here $\dot{\varepsilon}_{\max }$, i.e. $\approx 2 \pi f \varepsilon_{\max }$ which would lead to an horizontal shift of about $0.2 \mathrm{~s}^{-1}$. This is not enough to explain the significant differences observed in Fig. 10(b).

- The strain amplitude sensitivity. The strain varies along the sample length and during the test from 0.01 to $0.35 \%$, which means that the following test is close to DMTA in term of deformation amplitude $(0.1 \%)$ but somewhere between hydraulic machine tests and SHPB tests in term of strain-rates. The potential impact of the strain amplitude on the storage modulus variation, especially at low strains, is not clear and could introduce some variation of storage modulus simply due to strain amplitude variations. This point can affect the global shape of the master curve.

- Limitation of a 1D approach. It has been observed that the temperature was not perfectly uniform over a material section (see supplementary data) due to both higher heat losses at the edges and unsymmetrical strain localization. It seems important to investigate how such an approach could be extended to a 2D case which would not require this assumption. The present authors are currently working on a strategy based on the use of the Virtual Fields Method and a subset-based equilibrium to overcome such an issue.

While the current article focuses on a rather simple application (a homogeneous rectangular specimen in the elastic regime) as a first demonstrator, the present strategy has great potential for a range of problems not addressed with current test methods.

1. Fracture of brittle materials at high strain-rate. Brittle materials like glass or concrete are notoriously difficult to test at high rates of strain. A preliminary numerical simulation on glass with a $\pm 60 \mu \mathrm{m}$ excitation amplitude (limit of the current ultrasonic excitation system), and considering a very low damping coefficient of $\beta \approx 10^{-9}$ leads to a maximum stress of $\sigma=$ $\pm 95 M P a$, i.e. about the fracture stress of glass. As the material is not damped, the actuator can work over its whole displacement range to reach the failure stress of glass. Combining such test with a 2D generalization of the method would allow characterizing glass failure at high strain-rates. An initial experimental proof of principle has been conducted and a glass specimen has been successfully fractured.

2. Transverse fracture of composites. Because of the very low failure stress of a unidirectional (UD) composite in the direction transverse to the fibres, its tensile fracture stress is very difficult to obtain using SHPB experiments. A preliminary finite element study showed that an amplitude of $\pm 100 \mu \mathrm{m}$ would be required to reach $\sigma=50 \mathrm{MPa}$ with $\mathrm{E}=10 \mathrm{GPa}$ and $\beta \approx$ $10^{-8}$. While this is larger than what the current system used here can provide, it is not impossible to reach. An alternative could also be to fix a mass at the free end, this would however require a significant numerical test design campaign. The situation is even more difficult for the through thickness tensile properties where very small specimens have to be used. In this case, a thin laminate could be sandwiched between two steel blocks 
and displacement measurements just performed on the steel blocks with a direct reading to the transverse stress from the free end steel block.

3. Adhesives. The same idea as for the through-thickness composites test could be employed for adhesives which are notoriously difficult to test at high rates [39].

4. Yielding of engineering alloys. From initial finite element simulations, the actuator would also need an amplitude of $\pm 160 \mu \mathrm{m}$ to yield an aluminium sample (with $\sigma_{y}=280 \mathrm{MPa}, \mathrm{E}=72 \mathrm{GPa}$ and $\beta \approx 10$ $\left.{ }^{-10}\right)$. Again, this is too large for the current setup but a specific horn could be designed to boost the excitation amplitude further.

5. Extended strain-rate range. The current strain-rate range is imposed by the excitation frequency, the strain concentration within the sample and the covered range of temperatures (considering time-temperature superposition). It is possible to reach higher apparent strainrates by cooling down the sample prior to the start of the test, e.g. building a temperature controlled enclosure. According to preliminary calculations and tests, cooling the specimen down to $10^{\circ} \mathrm{C}$ only would allow reaching the equivalent of $10^{3} \mathrm{~s}^{-1}$ at room temperature, while cooling down to $-10^{\circ} \mathrm{C}$ would allow reaching $10^{4}$ $\mathrm{s}^{-1}$ at room temperature for PMMA samples. Working on sample geometry (using notches for instance) would lead to strain concentrations and thus higher local strain-rates. Nevertheless, the suitability of the low spatial resolution camera would need to be verified, and the 1D approach would most probably not be enough.

6. Heterogeneous materials. Although the present paper uses a simple homogeneous PMMA specimen for the sake of validation of the test concept, it is clear that the main asset of this image-based DMTA is to investigate heterogeneous materials. Indeed, while a technique such as DMTA is now well mastered and allows capturing the behaviour of rheologically simple materials over a very large range of strain-rates (see Fig. 10(b)), it cannot provide spatial maps of properties and therefore, cannot derive detailed properties for heterogeneous materials. An interesting case for instance could be injected polymeric sheets which have different properties in the skin than in the core. The present method has the potential to provide spatially-resolved properties.

These opportunities will be studied in more depth by the current authors in the near future.

\section{Conclusion}

The present work demonstrates the feasibility of a multiparametric identification on a single sample and falls within an effort to invent new high-strain test methodologies based on full-field imaging and inverse identification, to both overcome the limits of standard experimental strategies and take advantage of the deformation heterogeneities to achieve a full-characterization of a material from a "one-shot" test. It has been demonstrated that using a $20 \mathrm{kHz}$ high power ultrasonic excitation combined with infrared thermography and ultra-high speed imaging, a PMMA sample could be subjected to apparent strain-rates varying from less than $10^{0}$ $\mathrm{s}^{-1}$ to $10^{2} \mathrm{~s}^{-1}$ and temperatures varying from ambient to its $\mathrm{T}_{g}$. Within the studied strain-rate range, a significant increase in storage modulus has been evidenced that was not visible in the DMTA results. This interesting observation justifies the interest in developing new measurement techniques to capture the material behaviour at intermediate and high strain-rates. The validity of the approach has been checked using Finite Element modelling combined with synthetic grid image deformation. It demonstrates, under simple behaviour assumptions, that the error on identification is expected to remain below $10 \%$ over the sample length. This has been partially confirmed by the experimental data scatter. Finally, the potential of this new test technique has been underlined and it is expected that it will evolve to join the toolkit of researchers and engineers in mechanics of materials.

Acknowledgments This material is based on research sponsored by the Air Force Research Laboratory, under agreement number FA9550-15-1-0293. The U.S. Government is authorized to reproduce and distribute reprints for Governmental purposes notwithstanding any copyright notation thereon. The views and conclusions contained herein are those of the authors and should not be interpreted as necessarily representing the official policies or endorsements, either expressed or implied, of the Air Force Research Laboratory or the U.S. Government. The authors would like to thank Dr Jason Foley (AFOSR), Dr Matt Snyder (EOARD) and Dr David Garner (EOARD) for supporting this work.

Prof. Pierron acknowledges funding from EPSRC through grant $\mathrm{EP} / \mathrm{L} 026910 / 1$, as well as from the Wolfson Foundation through a Royal Society Wolfson Research Merit Award.

The authors are also grateful to Prof. Clive Siviour from the Department of Engineering Science at the University of Oxford for providing the DMTA results and for helpful discussions.

The authors are also grateful to Dr. Frances Davis from the Engineering and the Environment research group from the University of Southampton for performing the quasi-static tests, density measurements and DSC analysis on PMMA samples, as well as for helpful discussions.

All data supporting this study are openly available from the University of Southampton repository at http://doi.org/10.5258/SOTON/D0207.

Open Access This article is distributed under the terms of the Creative Commons Attribution 4.0 International License (http:// creativecommons.org/licenses/by/4.0/), which permits unrestricted use, distribution, and reproduction in any medium, provided you give appropriate credit to the original author(s) and the source, provide a link to the Creative Commons license, and indicate if changes were made. 


\section{Appendix}

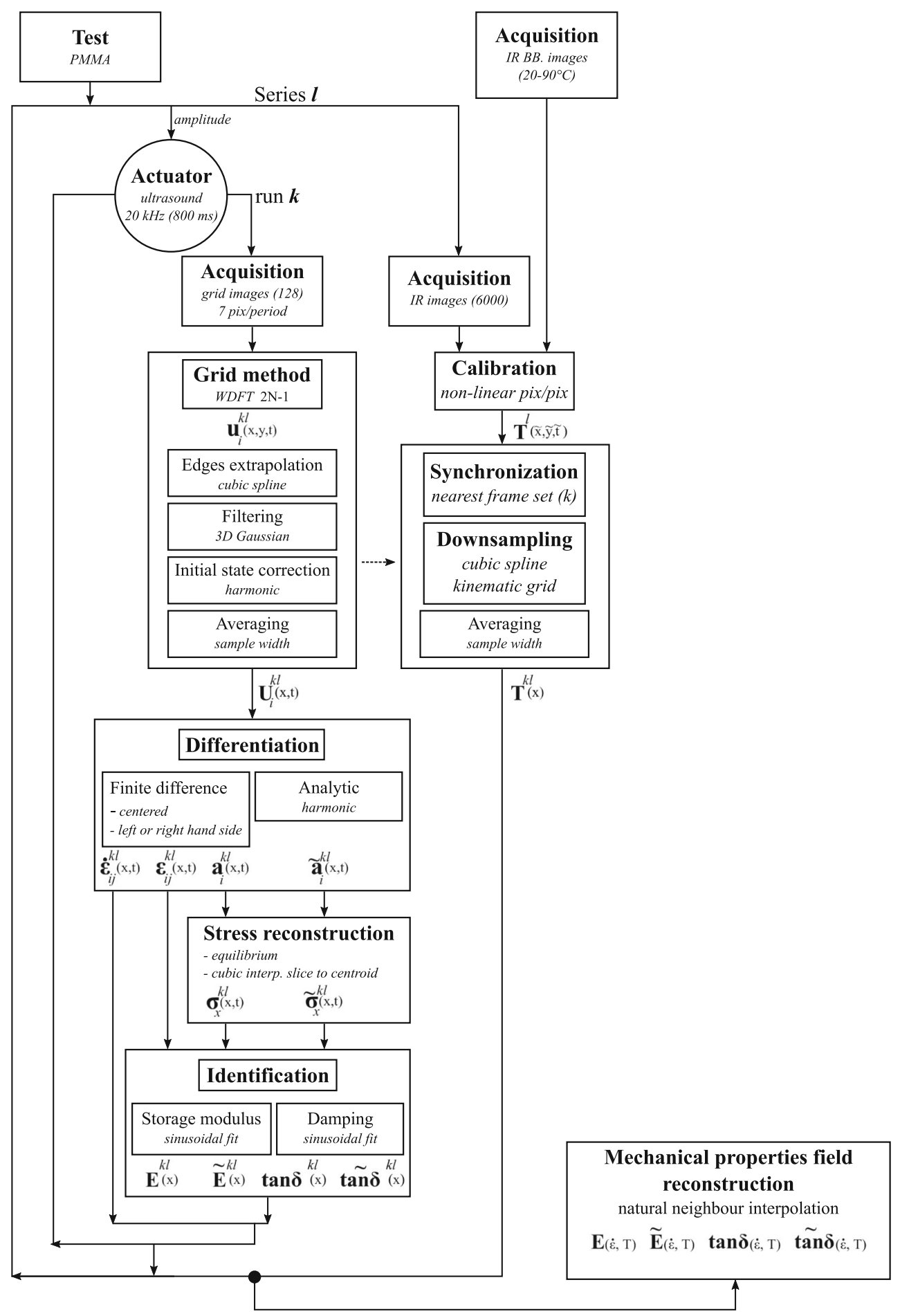

Fig. 16 Data post-processing procedure 


\section{References}

1. Capodagli J, Lakes R (2008) Isothermal viscoelastic properties of PMMA and LDPE over 11 decades of frequency and time: a test of time-temperature superposition. Rheol Acta 47(7):777-786

2. Crooks J, Marsh B, Turchetta R, Taylor K, Chan W, Lahav A, Fenigstein A (2013) Kirana: A solid-state megapixel ucmos image sensor for ultrahigh speed imaging. In: Proceedings of SPIE - the international society for optical engineering, volume 8659

3. Dreuilhe S, Davis F, Siviour CR, Pierron F (2016) Image-based inertial impact tests on an aluminum alloy. In: Conference proceedings of the society for experimental mechanics series, volume 3, pages 219-223

4. Dreuilhe S, Pierron F (2015) Extension of the non-linear virtual fields method to inertial heterogeneous high strain rate tests. Conference Proceedings of the Society for Experimental Mechanics Series 4:83-87

5. Field JE, Walley SM, Proud WG, Goldrein HT, Siviour CR (2004) Review of experimental techniques for high rate deformation and shock studies. Int J Impact Eng 30(7):725-775

6. Fruehmann R, Crump D, Dulieu-Barton J (2013) Characterization of an infrared detector for high frame rate thermography. Meas Sci Technol 24(10): 105403

7. Giraudeau A, Pierron F, Guo B (2010) An alternative to modal analysis for material stiffness and damping identification from vibrating plates. J Sound Vib 329(10):1653-1672

8. Grédiac M, Fournier N, Paris PA, Surrel Y (1998) Direct identification of elastic constants of anisotropic plates by modal analysis: Experimental results. J Sound Vib 210(5):643-659

9. Grédiac M, Paris PA (1996) Direct identification of elastic constants of anisotropic plates by modal analysis: Theoretical and numerical aspects. J Sound Vib 195(3):401-415

10. Grédiac M, Sur F, Blaysat B (2016) The grid method for in-plane displacement and strain measurement: A review and analysis. Strain 52(3):205-243

11. Halary J, Oultache A, Louyot J, Jasse B, Sarraf T, Muller R (1991) Viscoelastic properties of styrene-co-methyl methacrylate random copolymers. J Polym Sci B Polym Phys 29(8):933-943

12. Kendall MJ, Siviour CR (2015) Experimentally simulating high rate composite deformation in tension and compression: polymer bonded explosive simulant. J Dyn Behav Mater 1(2):114-123

13. Koohbor B, Kidane A, Lu W-Y (2016) Characterizing the constitutive response and energy absorption of rigid polymeric foams subjected to intermediate-velocity impact. Polym Test 54: $48-58$

14. Koohbor B, Kidane A, Lu W-Y (2016) Effect of specimen size, compressibility and inertia on the response of rigid polymer foams subjected to high velocity direct impact loading. Int J Impact Eng 98:62-74

15. Koohbor B, Kidane A, Lu W-Y, Sutton MA (2016) Investigation of the dynamic stress-strain response of compressible polymeric foam using a non-parametric analysis. Int J Impact Eng 91:170182

16. Koohbor B, Kidane A, Lu W-Y, Sutton MA (2016) Investigation of the dynamic stress-strain response of compressible polymeric foam using a non-parametric analysis. International Journal of Impact Engineering

17. Koohbor B, Mallon S, Kidane A, Lu W-Y (2015) The deformation and failure response of closed-cell pmdi foams subjected to dynamic impact loading. Polym Test 44(0):112-124

18. Lakes RS (1998) Viscoelastic solids, volume 9. CRC press
19. Le Louëdec G, Pierron F, Sutton MA, Siviour C, Reynolds AP (2015) Identification of the dynamic properties of al 5456 fsw welds using the virtual fields method. J Dyn Behav Mater 1(2):176-190

20. Li Z, Lambros J (2001) Strain rate effects on the thermomechanical behavior of polymers. Int J Solids Struct 38(20):35493562

21. Lukić B, Saletti D, Forquin P (2085) Use of simulated experiments for material characterization of brittle materials subjected to high strain rate dynamic tension. Philos Trans R Soc A Math Phys Eng Sci 375:2017

22. Moulart R, Pierron F, Hallett S, Wisnom M (2009) High strain rate photomechanics on composites: use of a ultra high speed camera and the virtual fields method. In: Rusinek PCA (ed) Workshop in memory of J.R. Klepasczko

23. Moulart R, Pierron F, Hallett SR, Wisnom MR (2011) Fullfield strain measurement and identification of composites moduli at high strain rate with the virtual fields method. Exp Mech 51(4):509-536

24. Mulliken A, Boyce M (2006) Mechanics of the rate-dependent elastic-plastic deformation of glassy polymers from low to high strain rates. Int J Solids Struct 43(5):1331-1356

25. Pierron F (2016) Addendum to 'Characterising the strain and temperature fields in a surrogate bone material subject to power ultrasonic excitation'. Strain 52(3):186-190

26. Pierron F, Forquin P (2012) Ultra-high-speed full-field deformation measurements on concrete spalling specimens and stiffness identification with the virtual fields method. Strain 48(5):388405

27. Pierron F, Zhu H, Siviour C (2014) Beyond Hopkinson's bar. Philos Trans R Soc A Math Phys Eng Sci 372(2023):20130195

28. Piro J-L, Grédiac M (2004) Producing and transferring lowspatial-frequency grids for measuring displacement fields with moiré and grid methods. Exp Tech 28(4):23-26

29. Reu PL, Miller TJ (2008) The application of high-speed digital image correlation. J Strain Anal Eng Des 43(8):673-688

30. Richeton J, Schlatter G, Vecchio K, Rémond Y, Ahzi S (2005) A unified model for stiffness modulus of amorphous polymers across transition temperatures and strain rates. Polymer 46(19):8194-8201

31. Robinson A, Dulieu-Barton J, Quinn S, Burguete R (2010) Paint coating characterization for thermoelastic stress analysis of metallic materials. Meas Sci Technol 21(8):085502

32. Rossi M, Lava P, Pierron F, Debruyne D, Sasso M (2015) Effect of dic spatial resolution, noise and interpolation error on identification results with the vfm. Strain 51(3):206-222

33. Rossi M, Pierron F (2012) On the use of simulated experiments in designing tests for material characterization from full-field measurements. Int J Solids Struct 49(3):420435

34. Seghir R, Witz J-F, Bodelot L, Charkaluk E, Dufrénoy P (2013) An improved lagrangian thermography procedure for the quantification of the temperature fields within polycrystals. Quantitative InfraRed Thermography J 10(1):74-95

35. Sutton MA, Orteu JJ, Schreier HW (2009) Image correlation for shape, motion and deformation measurements: Basic concepts, theory and applications. Image Correlation for Shape, Motion and Deformation Measurements: Basic Concepts, Theory and Applications, pages 1-321

36. Tochigi Y, Hanzawa K, Kato Y, Kuroda R, Mutoh H, Hirose R, Tominaga H, Takubo K, Kondo Y, Sugawa S (2013) A global-shutter cmos image sensor with readout speed of 1-tpixel/s 
burst and 780-mpixel/s continuous. IEEE J Solid State Circuits 48(1):329-338

37. Wang D, Lucas M, Tanner K (2013) Characterising the strain and temperature fields in a surrogate bone material subject to power ultrasonic excitation. Strain 49(5):409-419

38. Wang P, Pierron F, Rossi M, Lava P, Thomsen OT (2016) Optimised experimental characterisation of polymeric foam material using dic and the virtual fields method. Strain 52(1): 59-79
39. Yokoyama T, Nakai K (2015) Determination of the impact tensile strength of structural adhesive butt joints with a modified split hopkinson pressure bar. Int J Adhes Adhes 56:13-23

40. Yoon S-H, Giannakopoulos I, Siviour CR (2015) Application of the virtual fields method to the uniaxial behavior of rubbers at medium strain rates. Int J Solids Struct 69-70:553-568

41. Yoon S-H, Winters M, Siviour C (2016) High strain-rate tensile characterization of epdm rubber using non-equilibrium loading and the virtual fields method. Exp Mech 56(1):25-35 